\title{
Retrieval of agricultural crop height from space: a comparison of SAR techniques
}

\author{
Esra Erten ${ }^{a, *}$, Juan M. Lopez-Sanchez ${ }^{b}$, Onur Yuzugulluc ${ }^{c}$ Irena Hajnsek ${ }^{c, d}$ \\ ${ }^{a}$ Faculty of Civil Engineering, Istanbul Technical University, TR-34469 Istanbul, Turkey \\ ${ }^{b}$ Institute of Computing Research, University of Alicante, E-03080 Alicante, Spain \\ ${ }^{C}$ Institute of Environmental Engineering, ETH Zurich, $\mathrm{CH}-8093$ Zurich, Switzerland \\ ${ }^{d}$ Microwaves and Radar Institute, German Aerospace Centre (DLR), 82234 Oberpfaffenhofen, Germany
}

\section{Abstract}

This paper deals with the retrieval of agricultural crop height from space by using multipolarization Synthetic Aperture Radar (SAR) images. Coherent and incoherent crop height estimation methods are discussed for the first time with a unique TanDEM-X dataset acquired over rice cultivation areas. Indeed, with its polarimetric and interferometric capabilities, the TanDEM-X mission enables the tracking of crop height through interferometric SAR (InSAR), polarimetric interferometric SAR (PollnSAR) and the inversion of radiative transfer-based backscattering model. The paper evaluates the three aforementioned techniques simultaneously with a data set acquired in September 2014 and 2015 over rice fields in Turkey during their reproductive stage. The assessment of the absolute height accuracy and the limitations of the approaches are provided. In-situ measurements conducted in the same cultivation periods are used for validation purposes. The PollnSAR and morphological backscattering model results showed better performance with low RMSEs (12 and $13 \mathrm{~cm}$ ) compared to the differential InSAR result having RMSE of $18 \mathrm{~cm}$.

The spatial baseline, i.e. the distance between satellites, is a key parameter for coherent methods such as InSAR and PollnSAR. Its effect on the absolute height accuracy is discussed using TanDEM-X pairs separated by a baseline of $101.7 \mathrm{~m}$ and $932 \mathrm{~m}$. Although the InSAR based approach is demonstrated to provide sufficient crop height accuracy, the availability of a precise vegetation-free digital elevation model and a structurally dense crop are basic requirements for achieving high accuracy. The PollnSAR approach provides reliable crop height estimation if the spatial baseline is large enough for the inversion. The impact of increasing spatial baseline on the absolute accuracy of the crop height estimation is evident for both methods. However, PollnSAR is more cost-efficient, e.g. there is no need for phase unwrapping and any external vegetation free surface elevation data. Instead, the usage of a radiative traerties of the crops with consistent accuracy. The efficient retrieval of crop height with backscatnsfer based backscattering model provides not only crop height but also other biophysical proptering model is achieved by metamodeling, which makes the computational cost of backscattering inversion comparable to the ones of the coherent methods. However, effectiveness depends on not only the backscattering model, but also the integration of agronomic crop growth rules. Motivated by these results, a combination of backscattering and PollnSAR

${ }^{*}$ Corresponding author 
inversion models would provide a successful method of future precision farming studies.

Keywords: height estimation, TanDEM-X, rice, Synthetic Aperture Radar, PoISAR, interferometry, PollnSAR, Metamodel, Agriculture

\section{Introduction}

Vegetation height is a proxy descriptor for forest and crop inventories from which a number of other important attributes can be derived. In the context of sustainable environmental management, it is essential for characterising crops' phenological stages, yield, biomass and health, whereas in forestry applications it is a prerequisite for wood volume, carbon storage and biomass calculations. A conventional space-based height information is relevant for environmental applications, as it obriates the necessity for time consuming land-based surveys and provides detailed information about not only the height of the monitored vegetation, but also crops' biophysical attributes such as aboveground carbon storage and biomass (Minh et al., 2015). As one of the most popular microwave imaging techniques, space-based Synthetic Aperture Radar (SAR) with its all weather, day and night imaging capability, which is a particularly important factor for irrigated farming and rain forests, is of particular interest in environmental monitoring.

Compared to crops, forest height estimation with space-based SAR has been studied and discussed for different forest types in a variety of studies, which can -roughly- be categorised under three approaches: Radiative Transfer Theory (RTT), Interferometric SAR (InSAR) and Polarimetric Interferometric SAR (PollnSAR) (Guo et al., 2014; Karila et al., 2015; Li et al., 2014; Tanase et al., 2014). The limited use of space-based SAR for crop height estimation is due to the time interval between two stages of development, e.g. the morphological changes may occur in a week for cereals whereas in forestry it takes very long time. However, the re-visit time of the current generation of SAR satellites, which is 12 days for SENTINEL-1 and 11 days for TerraSAR-X, makes the detection of phenological changes in crop growth a possible task. Hence, there is a remarkable increase in the number of studies about space-based crop height monitoring applications with SAR images (Lopez-Sanchez et al., 2012; Rossi and Erten, 2015; Vicente-Guijalba et al., 2015; Yuzugullu et al., 2016b).

RTT in canopy modelling -dating back to 90's- has been used to detect biological and morphological changes of crops (Karam et al., 1992). RTT based models express the vegetation backscattering as a function of the canopy physical attributes such as plant height, stem width, number of leaves, leaf angle and leaf size, with assumptions on dielectric constant and ground scattering of the monitored target. Even though it is not easy to develop a direct relationship of the backscattering with biophysical parameters due to the complicated interaction between electromagnetic waves and vegetation canopy, interesting results have been obtained in terms of ground (Kim et al., 2013; Liu et al., 2016), airborne (Karam et al., 1995; Villard and Le Toan, 2015) and space (Inoue et al., 2014; Koay et al., 2007; Le Toan et al., 1997; Wang et al., 2009) based SAR measurements. In RTT based approaches, a heavy study with Monte Carlo (MC) simulations may be required to relate the observed backscattering to the entire canopy physical attributes. 
The powerful and well-known InSAR method, which is based on the evaluation of phase difference between two SAR acquisitions, has been applied to a wide range of forestry and land use applications. Its applicability, consistency, as well as its disadvantages and advantages, have been discussed in several articles, e.g. Bamler and Hartl (1998) and Hanssen (2001). However, considering the outdated space-based SAR sensors, crop height estimation with interferometry was not possible due to the low resolution and the large re-visit time, which causes unreliable phase information. In such a case, tandem interferometric acquisitions are more convenient to monitor crop height changes. Engdahl et al. (2001) and Srivastava et al. (2006) underlined the linear relationship between ERS 1/2 tandem coherences and the height of crops: the coherence decreases as the crop height increases. Recently, with significant improvements in space-based SAR sensors, Erten et al. (2015), Zalite et al. (2015) and Rossi and Erten (2015) showed that the TanDEM-X mission (bi-static interferometry) has a great potential in detecting crop height changes through its phenological development.

PollnSAR is another widely known advanced technique for deriving three dimensional (3D) vegetation products, which makes use of interferometry and polarimetry together to separate different scattering centres along the canopy. Its potential and limits have generally been shown through a variety of forests with air-borne SAR sensors (Cloude, 2010). PollnSAR forest height estimation from space has been recently discussed also with TanDEM-X images by Abdullahi et al. (2016), Lee and Fatoyinbo (2015) and Kugler et al. (2014). Nevertheless, when it comes to crop height estimation, the implementation of PollnSAR method is restricted to the air-borne acquisitions. Lopez-Sanchez et al. (2012) reported the first demonstration of the feasibility of PollnSAR to retrieve canopy height, whereas Pichierri et al. (2016) improved this demonstration by considering the vertical structure of the canopy and including multi-baseline data. Being a multipolarization interferometric technique, the challenge for crop height estimation by space-based PollnSAR is the requirement of large spatial baseline and high temporal resolution, which are explained in detail by Lopez-Sanchez and Ballester-Berman (2009).

The TanDEM-X satellite mission allows for the first time to assess these three crop height estimation methods in the context of operational monitoring, which was previously not possible due to the practical restrictions on temporal resolution time between satellites. TanDEM-X is an innovative SAR mission, first in space combining two twin satellites, particularly TerraSAR-X satellite (TSX-1) and another TerraSAR-X add-on satellite (TDX-1), to generate a global high-resolution digital elevation model (DEM) accomplishing HRTI-3 accuracy requirements by a bi-static configuration (Krieger et al., 2007). Although planned for global DEM generation, the orbit control, the spatial resolution and the polarimetric acquisition policy have led to new researches in space-based SAR applications, e.g Abdullahi et al. (2016), Mason et al. (2016), Rossi et al. (2016) and Zhang et al. (2016). Therefore, this paper explores the limitations and potentials of crop height monitoring by comparing, for the first time with a unique dataset, the three aforementioned techniques: RTT, InSAR and PollnSAR. Being one of the major crops consumed worldwide, paddy rice crop is chosen to assess crop height estimations from space. 
Here it should be noted that the performance of the SAR based crop height retrieval techniques is affected by the system frequency (Lopez-Sanchez and Ballester-Berman, 2009; Patel et al., 2006). In our case, the main purpose of the study is the assessment of the superficial plant height in an as accurate as possible way with the available space-based SAR data. For this reason, a short wavelength, providing enough backscattering from the vegetation volume, should be privileged due to the high extinction into canopy. The TanDEM-X system, working at X-band, has thus been favoured for this agricultural study, also taking into consideration its polarimetric and interferometric acquisition capability.

This paper continues with a concise theoretical background of RTT, InSAR and PollnSAR techniques for crop height estimation in Section 2. Section 3 involves the explanation of the study area and the TanDEM$X$ acquisitions. In Section 4, the implementation details of the theoretical approaches and crop height estimations are presented. Finally, Section 5 concludes the work with the discussion on the crop height retrieval methodologies in the context of agricultural studies.

\section{Methodical approaches for estimating canopy height with SAR imaging}

Let a dual-pol acquisition vector $\vec{k}=\left[\vec{k}_{1} \vec{k}_{2}\right]^{T}$ be a complex scattering vector that consists of two target vectors $\vec{k}_{1}=\left[S_{H H}^{1} S_{V V}^{1}\right]^{T}$ and $\vec{k}_{2}=\left[S_{H H}^{2} S_{V V}^{2}\right]^{T}$ obtained from multipolarization SAR images acquired at positions 1 and 2, respectively (Erten, 2013). Considering the TanDEM-X system, $\vec{k}_{1}$ and $\vec{k}_{2}$ correspond to measurements taken by the TSX-1 and TDX-1 satellites, which gather data at the same time and are separated by a baseline.

According to this acquisition geometry, the three basic approaches to crop height estimation from SAR are summarised below.

\subsection{RTT approaches}

Theoretical models based on RTT analyse the interaction of the electromagnetic waves with agricultural canopies, which are solved up to first order or beyond. In this study, to see the potential of the RTT models in crop height estimation, the model proposed by Karam et al. (1988) and developed by Le Toan et al. (1997) and Wang and Kong (2005) is implemented. Here, it should be noted that a limited number of morphology based rice scattering models (Liu et al., 2016; Wang et al., 2009; Zhang et al., 2014) have been developed over the past decade to characterise the canopy's geometric and physical structure. However, the morphological scattering model developed by Le Toan et al. (1997) is chosen due to its good agreement with X-band SAR images. Simulation errors less than $\sim 1 \mathrm{~dB}$ were reported (Inoue et al., 2014; Yuzugullu et al., 2016a).

This model provides a simplified description of plants architecture (stalks, tillers, leaves and panicles) and estimates the total backscattering $\left(\sigma^{0}\right)$ intensities from the rice canopy by considering the contribution from four physical scattering mechanisms $\left(S_{I}, S_{I I}, S_{I I I}\right.$ and $\left.S_{I V}\right)$ as visualised in Figure 1 and formulated in (1): 
$S_{I}$; Direct scattering from canopy

$S_{I I}$; Scattering from canopy followed by reflection from ground

$S_{I I I} ;$ Reflection from ground followed by scattering from canopy

$S_{I V}$; Reflection from ground followed by scattering from canopy again followed by reflection from ground.

These first order scattering mechanisms contribute to the backscattering signatures depending on the morphological structure of the canopy and the sensor parameters such as polarisation, wavelength and incidence angle of the incoming electromagnetic wave.

Given all the ground and vegetation descriptive parameters, the RTT based approaches can be used to estimate the total backscattering coefficient $\left(\sigma^{0}\right)$ for all the linearly polarised observations, e.g $\mathrm{HH}, \mathrm{VV}$, $\mathrm{HV}$ and $\mathrm{VH}$, by computing the ratio between the amplitudes of the scattered $\left(E^{s}\right)$ and incident $\left(E^{i}\right)$ electrical waves

$$
\begin{aligned}
E_{q}^{s}\left(\mathrm{r}^{\prime}\right) & =\frac{e^{i k r}}{r}\left(S_{I}+S_{I I}+S_{I I I}+S_{I V}\right) E_{p}^{i} & \\
\sigma_{q p}^{o} & =\frac{4 \pi r^{2}}{A_{i}} \frac{\left\langle\left|E_{q}^{s}\right|^{2}\right\rangle}{\left|E_{p}^{i}\right|^{2}}, & p, q=\{H, V\}
\end{aligned}
$$

where $r^{\prime}$ is the location of the scattering element, $A_{i}$ is the illuminated area, $k$ is the free space wave number and $r$ is the distance between the sensor and the target (Yuzugullu et al., 2015).

Morphology based RTT backscattering models requiring MC simulations, like (1), are computationally expensive due to a large number of scatterers per resolution cell $\left(\sigma^{0}\right)$. This leads to an underdetermined inversion problem with long computation time. To tackle this problem, metamodels, representing the input (rice biophysical parameters) and output $\left(\sigma^{0}\right)$ relations with approximated mathematical relation, may be performed (Sudret, 2015). The use of metamodels, which are employed to build domain specific solutions, overcomes the high computation cost, especially in case of physical parameter optimisation from backscattering observation (Vasko et al., 2011; Villard et al., 2015; Yuzugullu et al., 2016b).

According to the dual-pol acquisition vector of TanDEM-X, for any time $t$, there are four recorded copolarized backscattering channels. As each polarisation includes two acquisitions (one from TSX-1 and one from TDX-1), averaging co-polar backscattering values is convenient in order to decrease the noise by a factor of $\sqrt{2}$.

\subsection{InSAR approaches}

InSAR exploits the phase difference between two same polarised acquisitions to estimate the height of the scattering phase center (Hanssen, 2001). In contrast with RTT based approaches, the interferometric phase has direct relationship with the crop height.

The interferometric phase $\phi_{i n t}$, also called interferogram, is generated by the complex conjugate multiplication of two coregistered SAR images acquired at slightly different positions. $\phi_{\text {int }}$ is directly related 
to the height of scattering phase center and its quality is assessed with absolute value of complex correlation between acquisitions, also called coherence (Bamler and Hartl, 1998).

Since phase is measured module $2 \pi$, a parameter called height of ambiguity $(\mathrm{HoA})$, can be derived by computing the height difference corresponding with a $2 \pi$ phase shift:

$$
h_{2 \pi}=\frac{\lambda R \sin \theta}{B_{\perp}} .
$$

In (2), $\lambda, R, \theta$ and $B_{\perp}$ represent the wavelength, the geometric distance between satellite and target, the incidence angle and the perpendicular baseline between two acquisitions, respectively (Bamler and Hartl, 1998). The smaller $h_{2 \pi}$, the more accurate height estimation is.

According to the dual-pol acquisition vector of TanDEM-X, for any time $t$, for each polarisation there is one interferometric phase indicating the crop height. Since the location of the phase centre changes according to the density and morphological properties of the canopy as well as its interaction with electromagnetic wave, interferometric measurements from different polarisations may have discrepancies (Erten et al., 2015; Srivastava et al., 2009). It has to be noticed that the phase difference between polarimetric channels provides information about dielectric and geometric properties of the scattering medium. In contrast, the phase difference of the same polarised channels is only proportional to the crop scattering height.

\subsection{PollnSAR approaches}

The PollnSAR-based canopy height estimation has been used in the last decade with a special emphasis on forestry applications (Cloude, 2010). As summarised in Figure 1, these approaches employ a model of vegetation scenes formed by two layers: vegetation volume and ground surface. The scattering from ground is localised at a single point in the vertical coordinate, whereas the scattering from volume is described by a scattering function $f(z)$. Starting from this assumption, it is possible to express the interferometric coherences $\tilde{\gamma}_{v o l}\left(\kappa_{Z}, \vec{w}\right)$ that are measured at different polarimetric channels $\vec{w}$ as a function of the interferometric vertical wave number $\kappa_{Z} . \kappa_{Z}$ depends on the interferometric imaging geometry, and for a bistatic acquisition its relation with $h_{2 \pi}$ given in (2) is represented as

$$
\kappa_{Z}=\frac{2 \pi}{h_{2 \pi}} .
$$

The most complete expression for a bistatic interferometric system, considering that the response from the ground can be composed of two contributions (surface or direct scattering, and double-bounce scattering), is the following (Ballester-Berman and Lopez-Sanchez, 2011; Kugler et al., 2014; Treuhaft et al., 1996; Treuhaft and Siqueira, 2000):

$$
\tilde{\gamma}_{v o l}\left(\kappa_{Z}, \vec{w}\right)=e^{i \phi_{0}} \frac{\tilde{\gamma}_{V}+m_{D}(\vec{w})+\frac{\sin k_{z} h_{v}}{k_{z} h_{v}} m_{D B}(\vec{w})}{1+m_{D}(\vec{w})+m_{D B}(\vec{w})}
$$

where $\phi_{0}$ is the interferometric phase corresponding to the ground surface; $m_{D}(\vec{w})$ and $m_{D B}(\vec{w})$ are the ground-to-volume backscatter ratios corresponding to the direct $D$ and double-bounce $D B$ contributions; 


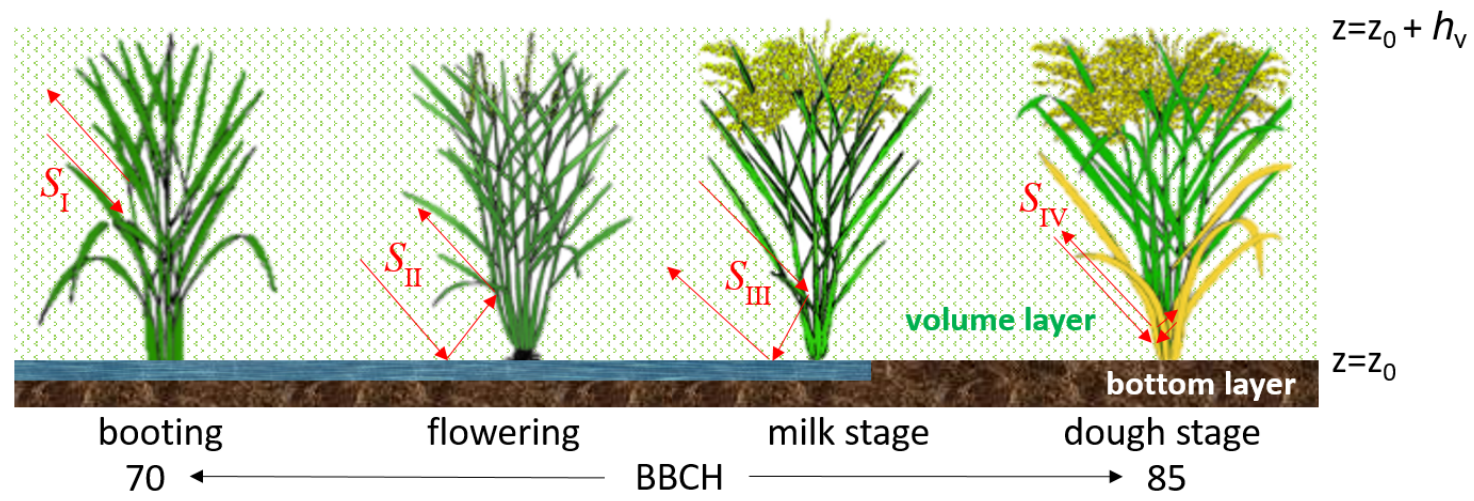

Figure 1: The $\mathrm{BBCH}$-scale identifying the phenology of plants in a standardised system and morphological structure information of rice plants during the acquisition time. $S_{I}, S_{I I}, S_{I I I}$ and $S_{I V}$ show major scattering mechanisms used to estimate the total backscattering $\sigma^{0}$ as in (1).

and $h_{v}$ is the vegetation height. The first term in the numerator, $\tilde{\gamma}_{V}$, is the coherence that would produce the volume alone (without the presence of the ground), which can be expressed as:

$$
\tilde{\gamma}_{V}=\frac{\int_{0}^{h_{v}} f(z) e^{i K_{Z} z} d z}{\int_{0}^{h_{V}} f(z) d z} .
$$

A note of caution is necessary for equation (4). The $\sin (x) / x$ term that appears before the doublebounce ground-to-volume ratio in the numerator is an extra decorrelation term present whenever a bistatic configuration is used. It is important to clarify that it depends on $k_{z}$, not on $\kappa_{Z}$ as was wrongly stated in (Kugler et al., 2014). This parameter is defined as (see Ballester-Berman and Lopez-Sanchez (2011); Treuhaft and Siqueira (2000) for details):

$$
k_{z}=\kappa_{Z} \sin ^{2} \theta_{0} .
$$

In the case of rice, and especially when plants are developed, the dominant scattering contribution from the ground is expected to be the double-bounce from the interaction between stems and the flooded soil, so equation (4) can be simplified as:

$$
\tilde{\gamma}_{v o l}\left(\kappa_{Z}, \vec{w}\right)=e^{i \phi_{0}} \frac{\tilde{\gamma}_{V}+\frac{\sin k_{z} h_{v}}{k_{z} h_{v}} m_{D B}(\vec{w})}{1+m_{D B}(\vec{w})}
$$

The scattering function $f(z)$ can be expressed in different ways according to different models or approximations of the scattering properties of the vegetation volume. It is well known that in agricultural crops the attenuation produced by the propagation of the waves through the vegetation volume depends on the polarimetric channel, being larger for vertical polarisation than for horizontal polarisation due to the predominant vertical orientation of the plant structural elements. In such a case, the formulation that takes this into account leads to the so-called oriented volume over ground (OVoG) model, which depends on the two extinction coefficients (vertical and horizontal). However, when this dependence on polarisation is not strong, one can use a simpler model named random volume over ground (RVoG) in which extinction is 
assumed to not depend on polarisation. In our case, since the available data are dual-pol (not fully polarimetric), hence reducing the dimensionality of the observation space or input data, and we have observed that the measured coherences do not deviate much from the RVoG predictions, we will assume the RVoG model for the inversion. With such an assumption, the only dependence of coherence (7) on the polarimetric channels comes from the ground-to-volume ratio $m_{D B}(\vec{w})$. In addition, this dependence makes the possible coherences (as provided by the model) to lie along a line on the complex plane.

\section{Study area and dataset}

In order to show the feasibility and the operational capability of InSAR, PollnSAR and RTT model in crop height estimation, as a representative work, paddy-rice fields located in Ipsala (Turkey) were selected. The rice-cultivated fields in the test site are located along the Maritza river, which forms the border between Turkey and Greece, see Figure 2. Figure 7(a) shows the topography of the area covered by TanDEM-X pairs. The agricultural fields, depicting by the white transparent area in Figure 7(a), are located in a flat area, since less than a metre of height variation among them is measured. The flat topography and the closeness of the water source (rivers and lakes) make this area a suitable region for rice cultivation. The seeds are sowed by broadcasting once per year about the end of May and irrigated by streams mainly from Maritza river.

The SAR images -whose main interferometric parameters are summarised in Table 1- were acquired by the TDX-1 and TSX-1 satellites. The previous studies with TDX-1/TSX-1 pairs (Erten et al., 2015; Rossi and Erten, 2015) have shown that (bistatic) InSAR is suitable for a temporal trend analysis of crop height measurements. However, due to the absence of the same-year ground truth, the dataset was insufficient for a one-to-one methodological comparison. Nevertheless, the crop height measurements were in accordance with the crop growth dynamics: a monotonically increasing crop height trend until the late reproductive stage. In this paper, in order to perform a fair comparison among the aforementioned methods in terms of crop height estimation, the rice plants should be dense and tall enough and not too dry to fulfill the requirements of volumetric scattering (Lopez-Sanchez and Ballester-Berman, 2009). Therefore, TanDEMX images acquired in September 2014 and 2015 are explored in depth with ground surveys conducted in the same cultivation period.

Additionally, in order to derive the plant height from the InSAR measure, another TanDEM-X pair acquired on November 26, 2012, which is assumed to have no vegetation (representing bare soil), has been used. In SAR acquisitions, a higher incidence angle impacts on the sensitivity of the vertical profile (Lopez-Sanchez and Ballester-Berman, 2009; Srivastava et al., 2007). In our dataset, a short wavelength with flat incidence angle, is well suited for the crop surface height estimation, since it yields a limited penetration of the wave inside the vegetated volume. Despite the incidence angle is same for all the data in the stack, the perpendicular baseline is varying in time, resulting in heights of ambiguity ranging from $6.25 \mathrm{~m}$ to $52.8 \mathrm{~m}$ (Table 1) according to (2). The spatial baseline is one of the most critical system 

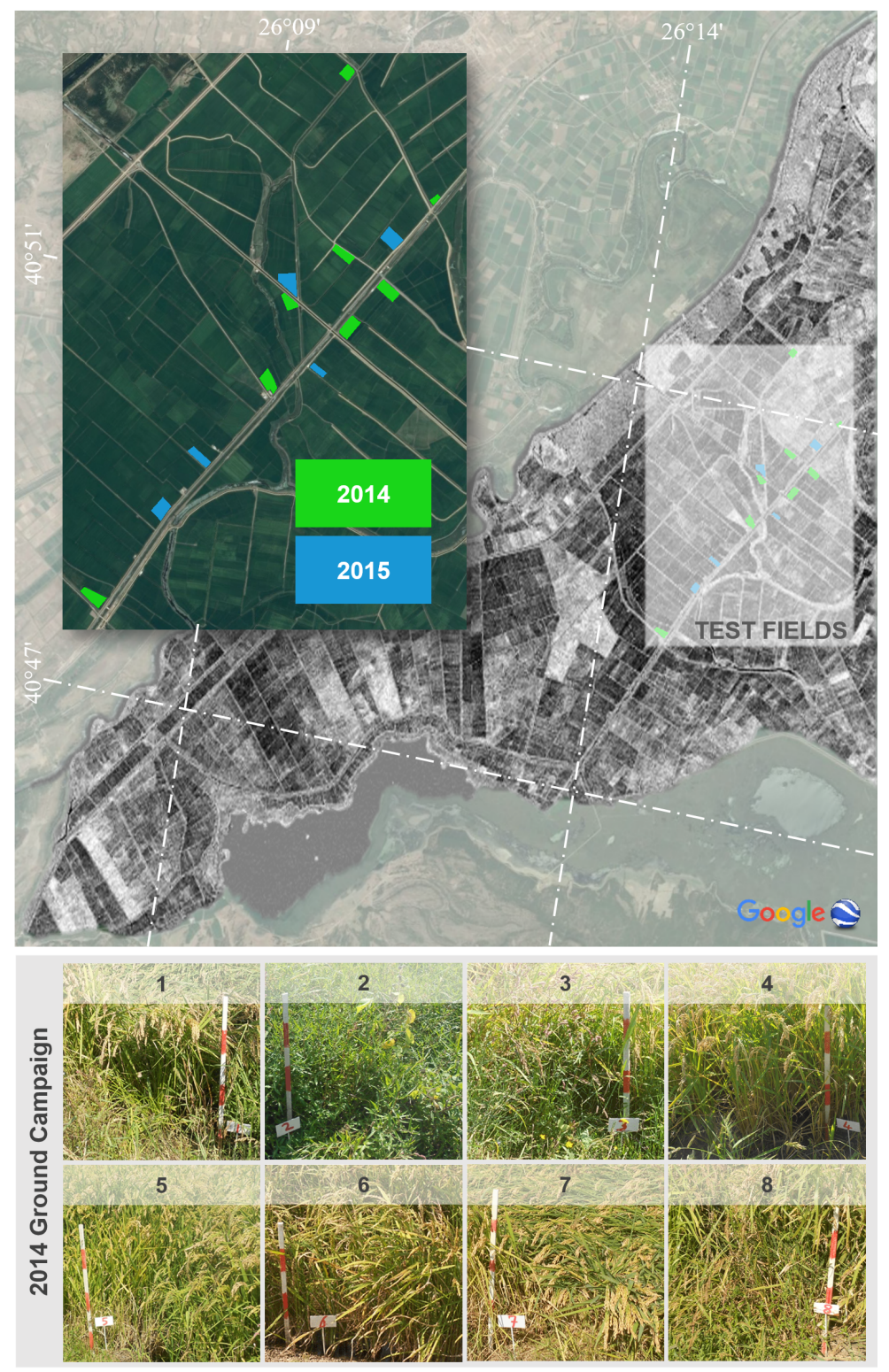

Figure 2: VV polarised TerraSAR-X acquisition taken on July 2, 2014, over the study area given by its amplitude image. The location of each test field analysed in this study is indicated by green and blue colours for 2014 and 2015 dataset, respectively. Photographs depicting the nature of paddy fields were shot in the day of the in-situ measurements conducted on September 11, 2014.

parameters of interferometric methods, since it drives the sensitivity of the measurements to the vertical distribution of elements in the scene.

Ground truth data for an entire rice cycle in 2014 were collected regularly, with an average temporal repetition about 11-days interval from June to October. The biophysical parameters such as stalk diameter, height above water, \#stalk $/ \mathrm{m}^{2}$, \#tiller/plant, \#leaf/stalk, leaf angle, stem angle, leaf length, leaf width, tiller length and plant phenological stage given by BBCH scale (Lancashire et al., 1991) were measured at four spatially independent points in each field, and averaged. The fieldwork for 2015 including measurements 
Table 1: Main Parameters of the Bi-static Interferometric Acquisitions

TanDEM-X pairs

\begin{tabular}{rccc} 
& 26.11 .2012 & 06.09 .2014 & 02.09 .2015 \\
\hline incidence angle [deg] & 36.8 & 36.8 & 36.8 \\
perpendicular baseline [m] & 181.3 & 101.7 & 932.0 \\
$\mathrm{HoA}[\mathrm{m}]$ & 32.3 & 52.8 & 6.25 \\
$\kappa_{Z}[\mathrm{rad} / \mathrm{m}]$ & 0.19 & 0.12 & 1.01 \\
crop condition & vegetation-free & reproductive stage & reproductive stage
\end{tabular}
Srivastava (2013).

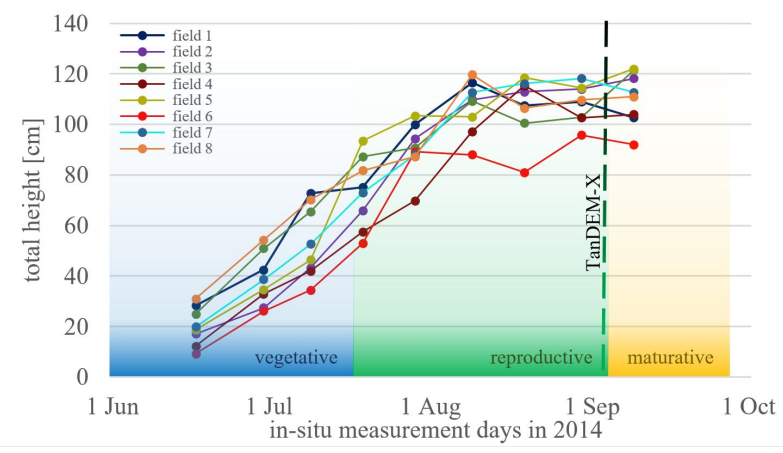

of stalk height, height above water, \#stalk/m², \#tiller/plant, water depth, leaf length, leaf width and \#tiller was carried out from June to September. As visualised in Figure 2, eight different test fields for 2014 and five different test fields for 2015 were selected. The measured crop heights for these test fields, which are varied in terms of size and heterogeneity, are shown in Figure 3. The plant heights reached their maximum after flowering; and afterwards they decreased due to water loss. On the acquisition dates, the entire test fields had reached their reproductive stage. This stage is defined in the range between $\mathrm{BBCH}$ values 53 (flag leaves are completely visible with $30 \%$ of the panicles emerged) and 77 (grains are in the late milk stage). Some of the fields were already in transition period to the maturation stage.

Here, it is worth mentioning that the number and the location of fields were identified by regional agricultural expertise by considering the discrepancy in harvest time. Since the crop height varies significantly within the fields $(\sim 10 \mathrm{~cm})$, comparing the crop height measurements between the averaged ground measurements and space-based crop height measurement per field is assumed to be sufficient. Readers interested in studies on ground truth planning for SAR acquisitions can refer to Patel and

Figure 3: In-situ crop height measurements conducted during the growth stages of rice in 2014 and 2015.

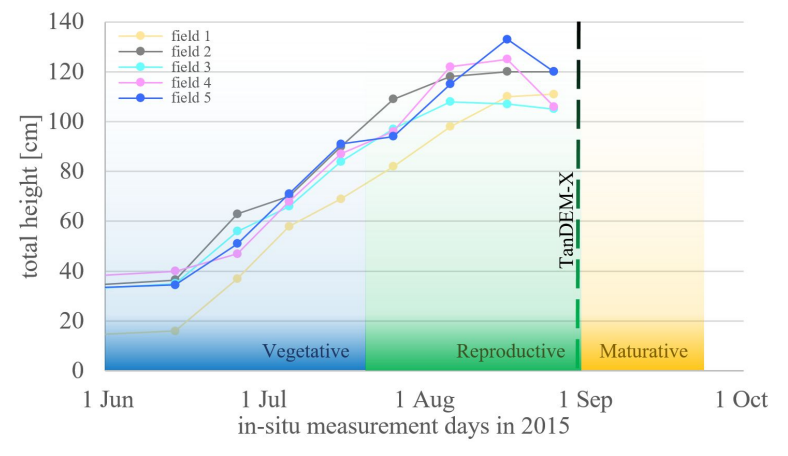




\section{Quality assessment of crop height from space: paddy-rice case}

For the crop height estimation with coregistered TanDEM-X pairs (CoSSC), the Interferometric TanDEMX Processor (ITP) at the German Aerospace Center (DLR) facilities has been employed (Rossi et al., 2012). The interferometric processor follows the standard processing stages. In particular, the principal steps are the interferogram generation and filtering, the phase unwrapping, the absolute phase retrieval and the geocoding. The same processor has been utilised for the previous rice crop height estimation studies with TanDEM-X (Erten et al., 2015; Rossi and Erten, 2015). More in detail, the multi-looking stage, implemented with a moving-average window of $9 \times 5$ samples in the range and azimuth directions, respectively, has been performed in order to obtain a spatial resolution of about $10 \mathrm{~m}$, which is sufficient considering the fields extension. Then, to help phase unwrapping for the acquisitions with a small height of ambiguity, the topographic phase has been removed before the unwrapping stage. No unwrapping errors have been detected. Finally, for the InSAR approach, all the DEMs have been generated over the same geographic grid and the absolute phase offset has been estimated with the help of an external Shuttle Radar Topography Mission (SRTM) DEM calibrated with IceSAT points (Rossi et al., 2016).

This section provides the implementation details of the aforementioned theoretical approaches and their results with the TanDEM-X dataset. The most straightforward way to evaluate the accuracy of crop height estimation approaches is a direct comparison with the reference in-situ data. For the September 6 , 2014, TanDEM-X acquisition, the crop heights interpolated from the ground campaign conducted on the September 1 and 11, 2014, were used as reference (Figure 3), whereas for the September 4, 2015, acquisition, the August 28, 2015, reference data has been employed. Accounting that the fields were in transition to the maturation stage, the 6-day difference between in-situ data and satellite image yields very small (nearly $1-2 \mathrm{~cm}$ ) height discrepancies between crops, thus not being a limiting factor in the accuracy assessment.

\subsection{RTT based crop height estimations with metamodeling}

Although the morphology-based electromagnetic scattering model in (1) can be used to simulate $\sigma^{0}$ for all possible polarisations and incidence angles for several frequencies, here, for time $t$, only $\mathrm{HH}$ and VV acquisitions are available for determining the whole crop parameters. To deal with this problem and to decrease the computational cost related to $M C$ simulations, the inversion is performed within a parameter space using a Polynomial Chaos Expansion (PCE) metamodel. PCE metamodel, which is well suited to inversion problems, is a widespread computational model for evaluating and replacing the original complex model by approximated model built from an experimental design (Rajabi et al., 2015; Sochala and Le Maitre, 2013; Sudret, 2015).

The key aspect of PCE is that if the distribution of $\xi$ is known, then the distribution of $Y$ can be estimated 
for any functions in the form of $Y=f(\xi)$. The PCE expands the function in a polynomial series as in (8):

$$
Y=f(\xi)=\sum_{j=0}^{\infty} a_{j} \Psi_{j}(\xi)
$$

Here, $\xi \in \mathfrak{R}^{M}$ is the random vector including whole morphological parameters (\#plants, \#tillers, stalk height and diameter, leaf width and length of each tiller, \#panicles) expressing backscattering $\left(\sigma^{0}\right) Y, a_{j} \in \mathfrak{R}$ and $\Psi_{j}(\xi) \in \mathfrak{R}$ are the coefficients and orthonormal basis of the polynomial series with respect to the distribution of $\xi$. In our problem, $f(\xi)$ represents the MC simulation model (1) given by Wang and Kong (2005). For practical reasons, it is necessary to truncate the representation (8) with limited number of expansions:

$$
Y \cong f(\xi)=\sum_{j=0}^{D} a_{j} \Psi_{j}(\xi) .
$$

In the current study, polynomial basis and coefficients are constructed using a non-intrusive least-angle regression-based method explained by Sudret (2015). Once built, PCEs are used instead of numerical simulation of the backscattering model which decreases simulation time drastically. In this study, PCE was implemented for (1) in MATLAB ${ }^{\circledR}$ within the UQLab framework by Marelli and Sudret (2014) and its performance was evaluated by comparing the surrogated backscattering intensity with that from TanDEM$X$ and Radarsat-2 images in Yuzugullu et al. (2016a) and Yuzugullu et al. (2016b), respectively. Here, it has to be noticed that the dimension of morphological parameter vector $\xi$ depends mainly on the type of EM model $f(\xi)$ used to represent the scattering phenomena. Metamodeling can be applied to any kind of scattering model (Koay et al., 2007; Liu et al., 2016) without considering its complexity, which is reduced by its surrogate substitutes.

Considering the presence of speckle noise and the ill-posed nature of the inverse algorithm, it is convenient to use a probabilistic optimisation algorithm. This study uses Particle Swarm Optimisation (PSO) in the space of the possible morphologies (Kennedy et al., 2001). The following fitness function (10) for $i^{\text {th }}$ iteration is employed:

$$
\min \mathbb{C}_{i}=\left(\sigma_{\mathrm{HH}}-\bar{\sigma}_{\mathrm{HH}_{i}}\right)^{2}+\left(\sigma_{\mathrm{VV}}-\bar{\sigma}_{\mathrm{VV}_{i}}\right)^{2}
$$

where $\left(\sigma_{\mathrm{pp}}\right)$ and $\left(\bar{\sigma}_{\mathrm{pp}}\right)$ shows the simulated and measured intensities for $\mathrm{HH}$ and $\mathrm{VV}$ channels.

The optimisation algorithm runs until it converges to a representative distribution with $n$ samples. Lastly, the median of the resulting distribution is used as the result of the fitness function.

The PSO algorithm requires the constraint boundaries for the crop biophysical descriptors. The boundaries of the biophysical parameters differ for each main growth stage, and, as a result, varying optimisation boundaries are needed to follow the crop growth dynamics. Required boundaries are provided using the ground measurements collected from the in-situ surveys conducted between the years of 2013 and 2015 in the study area. Within the applied methodology, high-dimensional parameter space -bounded by the growth data extremes- can easily be updated for different crop genotypes whenever new ground measurements are available. Boundaries of the morphological axes of the multi-dimensional 

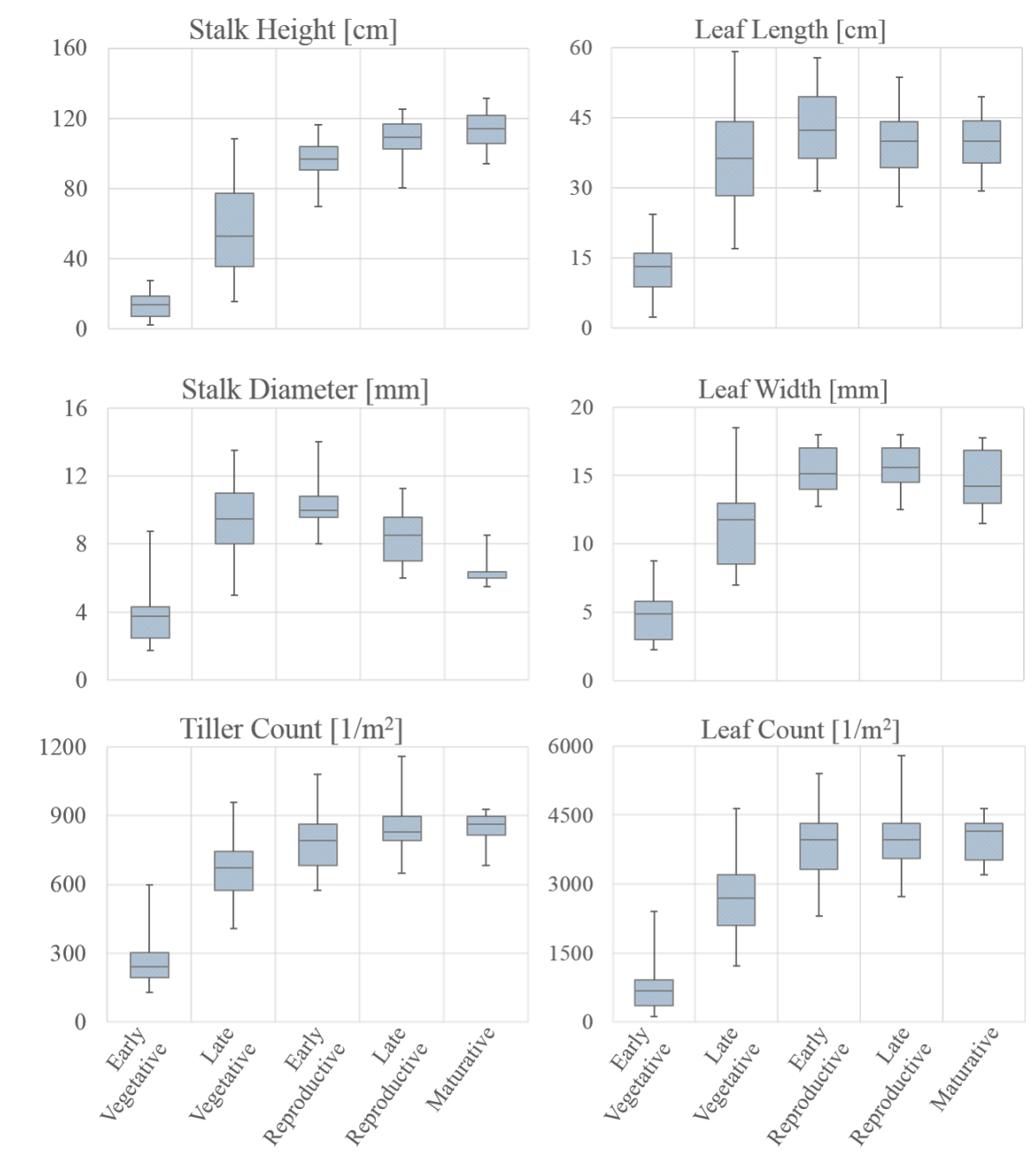

Figure 4: Box-whisker plot of rice morphological parameters from in-situ measurements conducted between 2013 and 2015 with respect to the rice's growth stages.

parameter space are given as a box-whisker plot in Figure 4 for five main growth stages of rice. To eliminate biologically impossible structures, e. g. $100 \mathrm{~cm}$ stalk height with $1 \mathrm{~cm}$ leaf length, PSO is constrained by convex hull, which takes into account biophysical and agronomical growth rules from literature and ground campaigns.

The stochastic optimization method aims to find a set of morphological vectors to obtain a complete crop morphology in the given bounded parameter space from the measured backscattering intensities in the observation space. The morphological vectors obtained from $n$ iterations are reported as a result. The presence of structural variance in the field and within the SAR data prevents from having a single optimum solution. Instead, the optimisation algorithm provides a distribution of each morphological parameter as an output. Details of the proposed approach are given in (Yuzugullu et al., 2016a,b).

Figure 5 summarises the mean and the standard deviation of the backscattering values in $\mathrm{dB}$ for each 


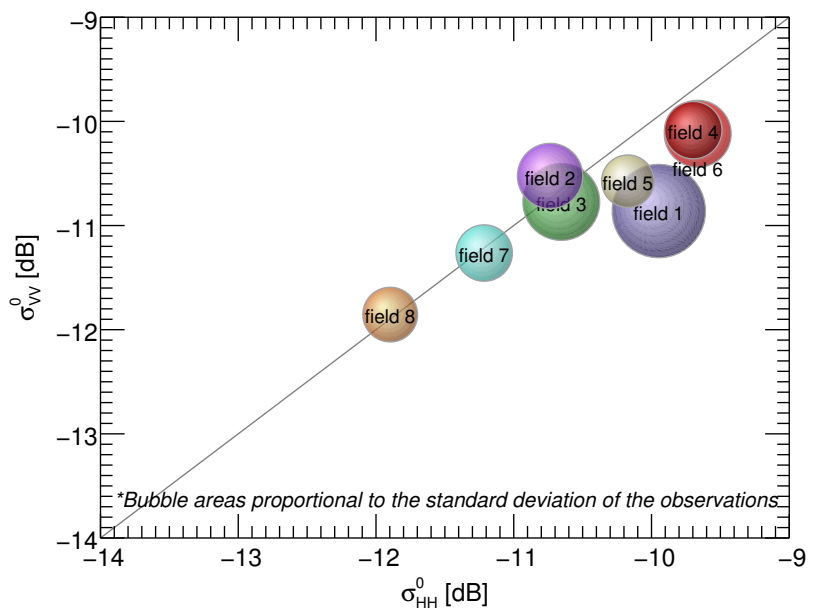

(a) 2014

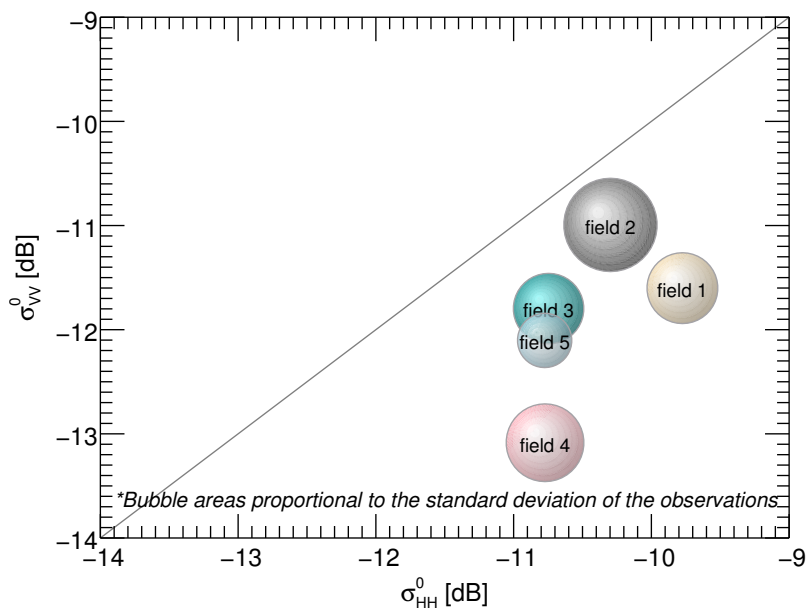

(b) 2015

Figure 5: Scatter-plot of the $\mathrm{HH}$ and VV mean intensity values transformed to $\sigma^{0}$ as observed by averaging the total number of pixels -given in Table 2- for each field for 2014 (a) and 2015 (b) dataset. The area of bubbles is proportional to the square root of the sum of the variance of the observations.

polarimetric acquisition, which are the only inputs of the observation space. In Figure 5, high variations in $\sigma^{0}$ values and their variances among the fields were observed, emphasising a high impact of smallscale morphological diversity on backscattering values. Additionally, sowing density and having different genotype with altering maximum leaf and tiller number can alter the plant morphology and lead to varying backscattering intensity.

Figure 6 gives an overview of the height measurements by PCE metamodel based RTT inversion algorithm, whereas Table 2 reports the descriptive statistics of the height measurements. In Figure 6, the ground-based crop height measurements are depicted as bullets. For the current analysis, $n$ is taken as 200. Therefore, at the end of the analysis, 200 possible rice crop physical structures for a specific backscattering intensity pair exist. The differences between iteration results -as seen in the box plot- are linked to the fact that RTT based modelling considers the full crop morphology and structural density of the crops, i.e., crops can be all in the same height but all with different possible other morphological parameters. This is the reason that -as wanted- PSO algorithm may converge to a different morphology vector in each iteration, causing variances exceeding $10 \mathrm{~cm}$ for some fields.

In Table 2, $h_{R T T}$ and $s_{h_{R T T}}$ specify the median and the sample standard deviation of the iteration results, where $\overline{\Delta h}$ gives their offsets from the height measurements obtained from ground surveys -entitled an absolute accuracy-. A first visual qualitative interpretation of the results clearly reveals that the crop heights can be estimated with minimum 3 and maximum $22 \mathrm{~cm}$ diversity from their actual value, which is of considerable agronomic interest. It can also be easily seen that the sample standard deviations obtained from the 200 iterations are quite low with a maximum for the field 5 in 2015 dataset with $s=9 \mathrm{~cm}$. Note that the absolute accuracy results $\overline{\Delta h}$ exhibit either positive or negative values. The main reason of such 


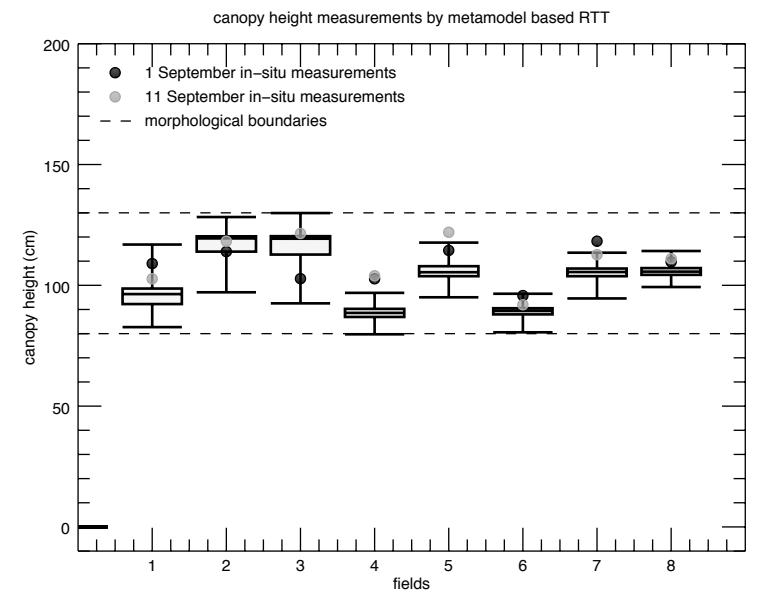

(a) 2014

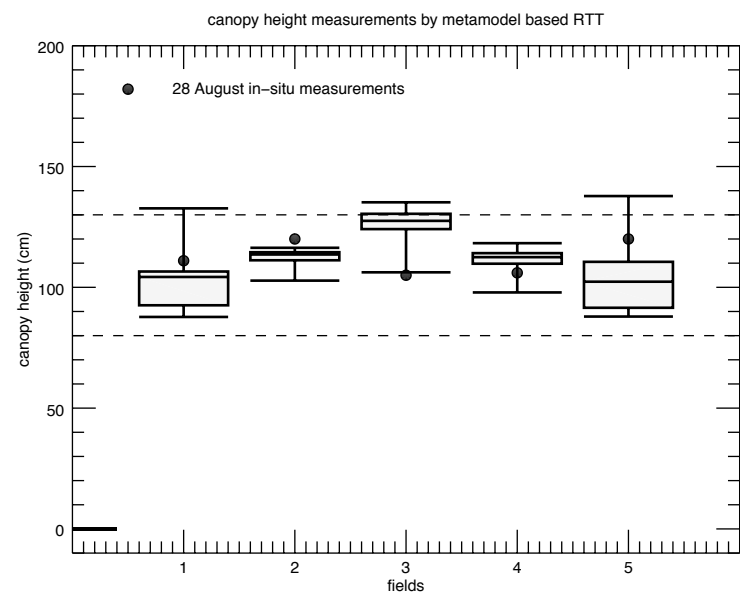

(b) 2015

Figure 6: 2014 (a) and 2015 (b) box-whisker plots of the set of all feasible canopy height measurements by PCE metamodel based RTT.

Table 2: Crop height estimations [cm] by PCE metamodel based RTT, using the combination of two polarisations

\begin{tabular}{|c|c|c|c|c|c|c|c|c|}
\hline \multirow[b]{2}{*}{ field \# } & \multicolumn{4}{|c|}{2014 [cm] } & \multicolumn{4}{|c|}{$2015[\mathrm{~cm}]$} \\
\hline & \# pixels & $h_{R T T}$ & $s_{h_{R T T}}$ & $\overline{\Delta h}$ & \# pixels & $h_{R T T}$ & $s_{h_{R T T}}$ & $\overline{\Delta h}$ \\
\hline 1 & 728 & 96 & 7 & 9 & 348 & 104 & 7 & 6 \\
\hline 2 & 1086 & 119 & 6 & -3 & 513 & 113 & 4 & 6 \\
\hline 3 & 1303 & 119 & 7 & -7 & 310 & 127 & 7 & -22 \\
\hline 4 & 972 & 88 & 6 & 14 & 292 & 112 & 4 & -6 \\
\hline 5 & 974 & 105 & 4 & 12 & 310 & 102 & 9 & 17 \\
\hline 6 & 501 & 89 & 3 & 4 & - & - & - & - \\
\hline 7 & 675 & 105 & 3 & 9 & - & - & - & - \\
\hline 8 & 909 & 105 & 2 & 4 & - & - & - & - \\
\hline
\end{tabular}

variation is related to the dynamics of the optimisation algorithm and the number of iterations, which have a direct impact on the resulting statistics. The absolute accuracy results from 2015 data set are in accordance with the previous year data set confirming the feasibility of the PCE metamodel based RTT approach in crop height monitoring.

\subsection{InSAR based crop height estimations}

To calculate the height of the crops, four interferograms (two bistatic acquisitions in two polarization) were generated. As shown in Figure 7(a), a complementary TanDEM-X pair acquired on November 26, 2012 was also processed by ITP in order to get the ground elevation of the fields (vegetation-free reference 
DEM).

After the surface elevation models had been generated, the elevation error maps presented in Figure $7(\mathrm{~b}-\mathrm{d})$ were derived by scaling the standard deviation of the interferometric phase $\sigma_{\phi_{\text {int }}}$ (calculated by (Hanssen, 2001, Eq. 4.2.27)) with the height of ambiguity; $h_{2 \pi}$. High values reflect the influence of decorrelation between TDX-1 and TSX-1 pairs, yielding less accurate heights, whereas low values reflect more reliable elevation measurements. The error of the interferometric measurements increases as the spatial baseline decreases. In addition to the sensor' geometry, the accuracy of the DEM depends also on the monitored target as well. For example, the elevation of surfaces covered by non-moving water cannot be usually derived with TanDEM-X due to the fact that water decorrelates in a fraction of second (Bamler and Hartl, 1998; Romeiser et al., 2014). In the 2015 acquisition (Figure 7(d)), flat terrain shows very low error values, with the exception of water covered areas. Moreover, distinct variations can be observed between agricultural and hilly areas. Compared to the 2015 acquisition, the error values decrease significantly for hilly areas, but slightly increase for agricultural areas in the 2014 acquisition (Figure 7(c)). A possible explanation is an increased volume decorrelation in the forest-covered hilly areas due to the very low $h_{2 \pi}$ of the 2015 acquisition. Volume decorrelation is not relevant factor for crops at this stage, where the reflection is mainly coming from the grains at the canopy top surface. Finally, a visual error map analysis of the 2012 acquisition compared to the 2014 one demonstrates that decreasing $h_{2 \pi}$ from $52.8 \mathrm{~m}$ to $32.3 \mathrm{~m}$ yields slightly better elevation accuracy.

Figure 8 summarises the elevation error of interferometric measurements for each test field. In agreement with Figure 7, the dependency of $h_{2 \pi}$ on the error plot is apparent. While the estimated elevation error is high in September 2014 images, the ones in November 2012 and September 2015 images are lower, changing between 8 and $43 \mathrm{~cm}$. It is to be noticed that even though the main error difference between images is due to the changing spatial baseline in time, the other causes of variation, such as those due to the different morphological properties of plants, remain, and their impact on the error analysis is evidenced by the coherence values -hence the standard deviation of interferometric phase. Appearance of panicles and an increase in leaf size at the late reproductive stage affect the attenuation of $\mathrm{HH}$ and VV polarised wave. In the maturation stage, attenuation decreases and gets similar for both polarisations.

Following the height-error analysis, the canopy heights were calculated by the surface elevation differences between the canopy (September) and ground (November). Assuming that the elevation information obtained from the November images gives the soil surface (bottom layer in Figure 1) and remains unchanged in time, the elevation differences are only caused by the canopy height variation.

For assessing the InSAR based crop height estimates, box-whisker plots in Figure 9, showing the height estimation values obtained for each pixel within the fields, were used. In Figure 9(a)-(b), the height measurements in 2014 are given for two co-polarised acquisitions for comparison purposes. Based on the comparison between interferometric measurements and in-situ measurements -indicated by the black and 


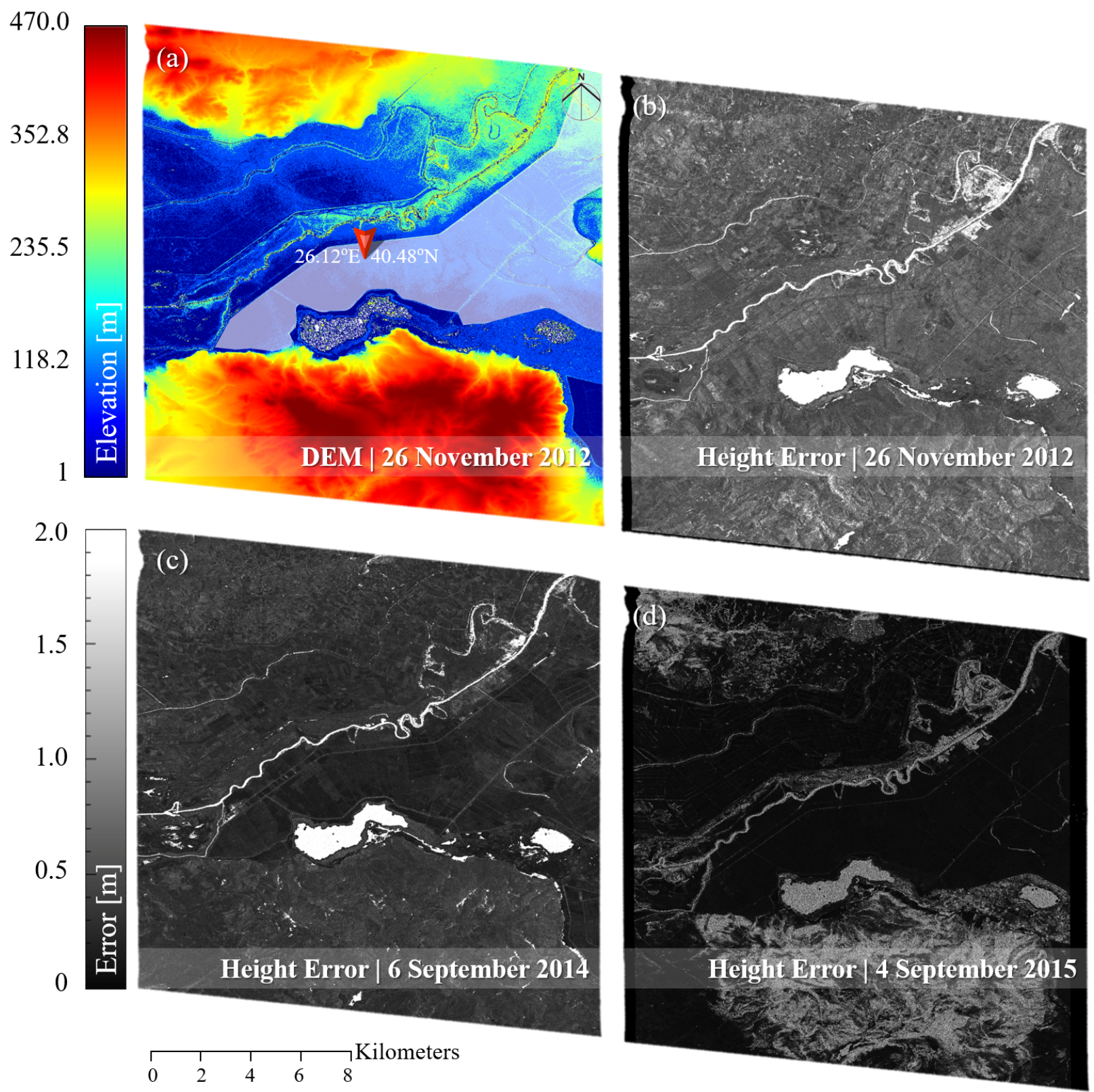

Figure 7: (a) Topography of the study area produced from TanDEM-X pairs acquired on the November 26, 2012. The white transparent area depicts the location of the agricultural fields shown in Figure (2). (b-d) Height error $\left(\sigma_{\phi_{\text {int }}} \frac{h_{2 \pi}}{2 \pi}\right)$ maps of the entire scenes from 2012 to $2015 \mathrm{HH}$ polarised acquisitions.

grey coloured circles in the figures-, two conclusions are clearly drawn: (i) polarisation diversity causes slight variations in the crop height estimation and (ii) it is necessary to distinguish between relative and absolute accuracy when interpreting the feasibility of TanDEM-X data in the context of InSAR based crop height monitoring. In detail, the relative height error (random noise) is derived from the coherence values, and depends on the system parameters (spatial baseline, wavelength and incidence angle) and the number of looks used in the processing as in Figure 7 and 8 . This is associated to the interferometric 


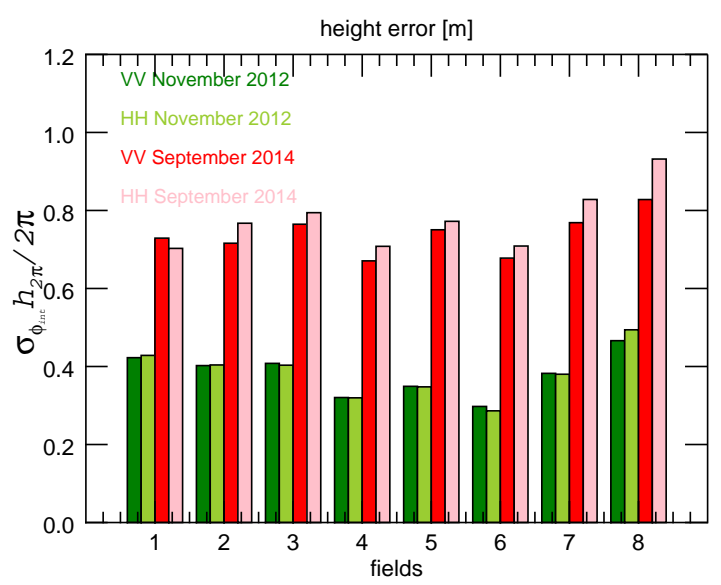

(a)

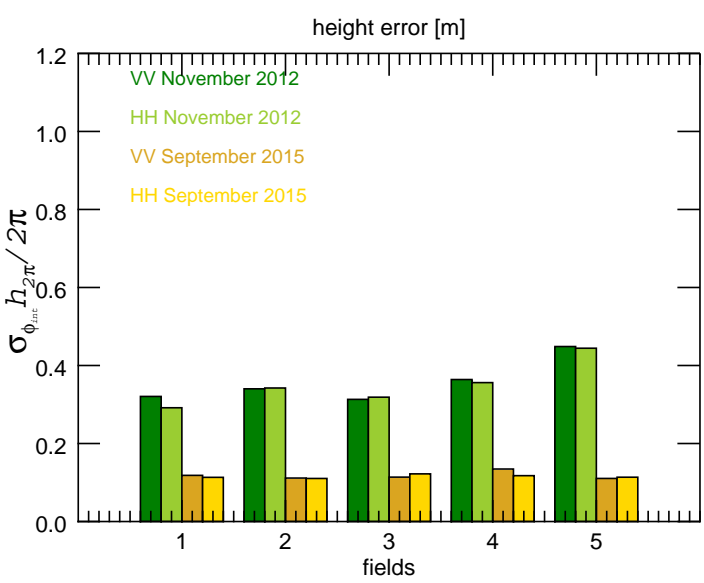

(b)

Figure 8: The mean interferometric height error for each test field in 2014 (a) and 2015 (b) as a function of the scaled HoA and the standard deviation of interferometric phase, which is directly related to the coherence (Hanssen, 2001, Eq. 4.2.27).

phase noise (Rizzoli et al., 2012). In contrast, the absolute accuracy refers to the real elevation discrepancy between the top of the canopy and the InSAR based crop height measure. The first conclusion has been shown by Erten et al. (2015) and Kugler et al. (2014) with different datasets experimentally, but not analytically due to the complex dynamic interaction between the electromagnetic wave and canopy. (Erten et al., 2015; Kugler et al., 2014).

The results visualised in Figure 9 are summarised in Table 3 and and 4. In the tables, the first two columns of $\mathrm{HH}$ and $\mathrm{VV}$ measurement results show the mean of the calculated height measurements and their standard deviation, which is the sum in quadrature of the variances of the interferometric measurements from vegetated and vegetation-free acquisitions. The third column $\overline{\Delta h}$ provides the mean height differences between the InSAR (the first column) and the ground survey based measurements. Within the results, it can be concluded that the measurements based on VV polarised acquisitions are more close to the top vegetation layer compared to the $\mathrm{HH}$ acquisitions due to their larger attenuation properties, making the absolute accuracy of the height measurements higher. Taken together with Figure 8 , these results reveal that having more reliable measurements (high coherence, low error) alone does not guarantee a more accurate height estimation. In other words, the stability of the phase center (i.e. relative error) is not linked to with its position (i.e. absolute error).

The morphological properties of crops lead not only to the deterioration of absolute accuracy of $\mathrm{HH}$ polarised InSAR based measurements, but also to discrepancies among the absolute accuracy of the fields even in the same polarisation. Considering both the polarisations in Table 3, it can be stated that the most reliable measurements do not always achieve better absolute accuracy than the less reliable measurements like field 7 and 8 , having the standard deviation of $95 \mathrm{~cm}$ and $108 \mathrm{~cm}$, respectively. The key issue of having highest absolute accuracy is related to the presence of dense vegetation. For example, on the date of the acquisition in 2014, the length of the leaves for field 1 and field 2 were smaller than the ones for field 7 


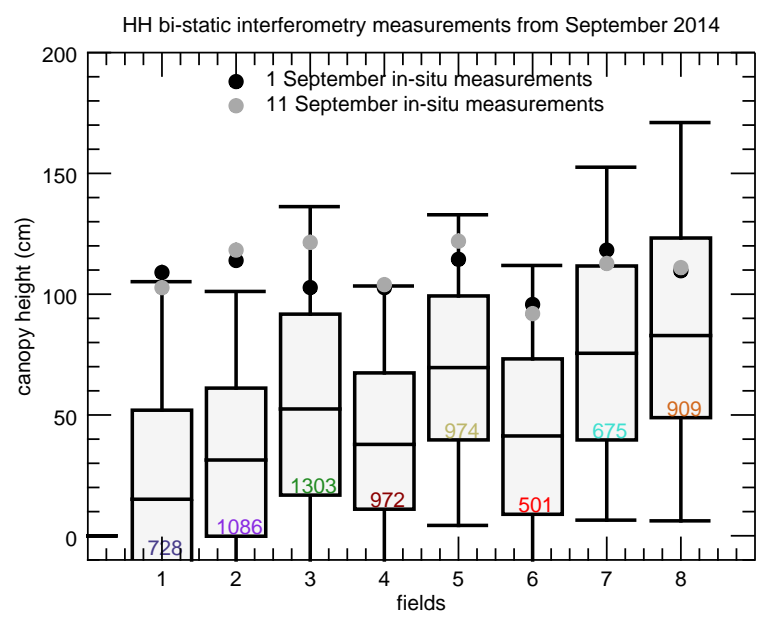

(a) 2014

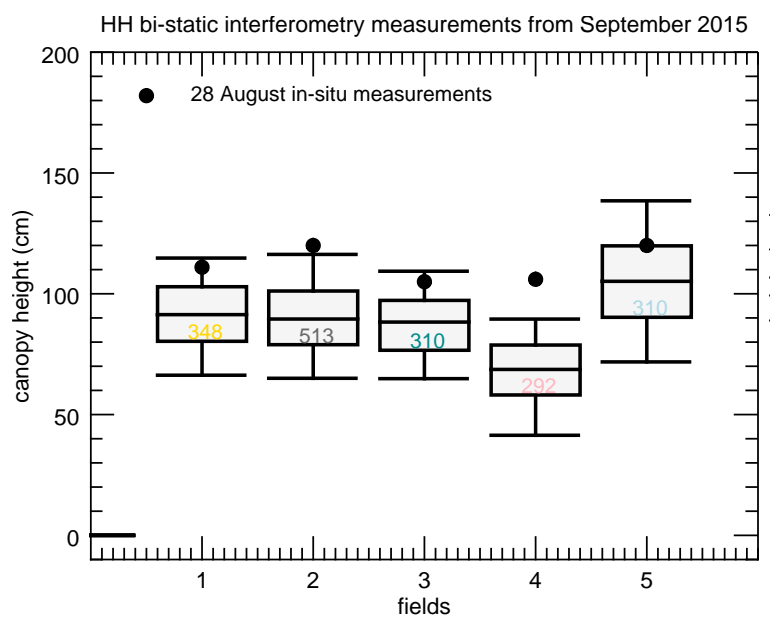

(c) 2015

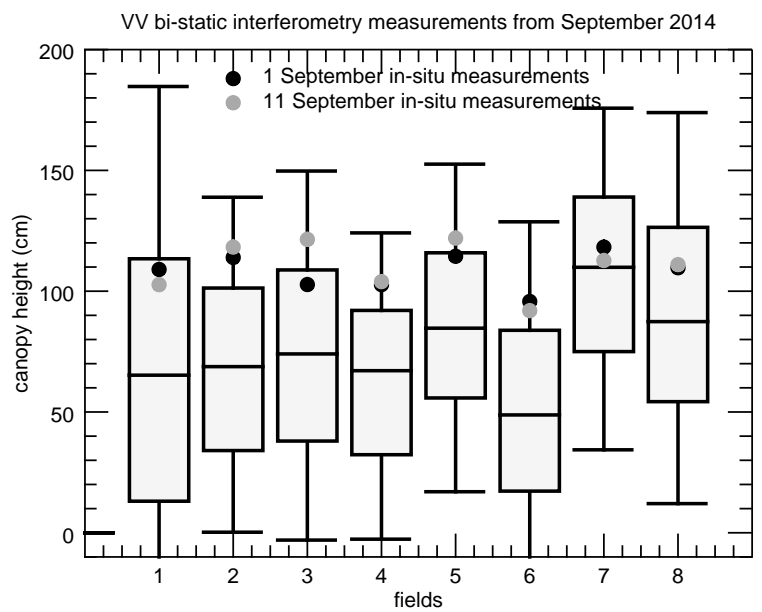

(b) 2014

VV bi-static interferometry measurements trom September 2015

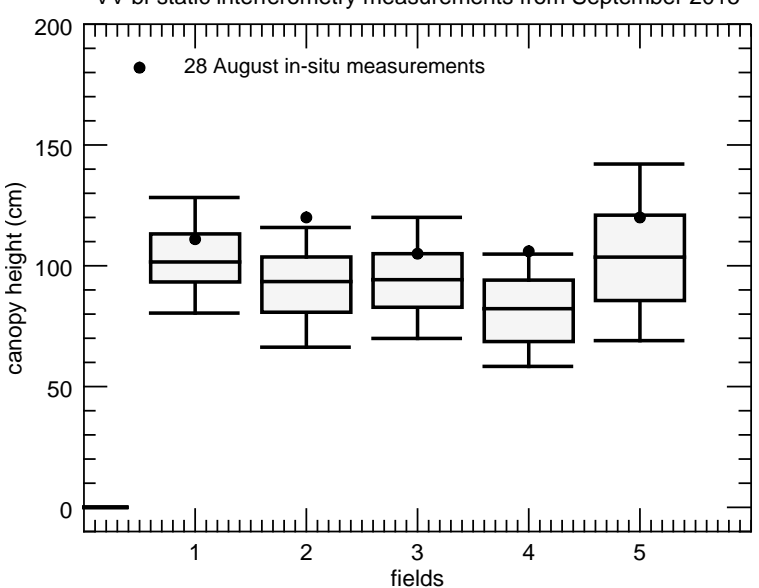

(d) 2015

Figure 9: Box-whisker plots of the canopy height measurements by InSAR-based approach for 2014 (a-b) and 2015 (c-d) dataset, given with conducted in-situ measurements. The numbers inside the box plots in (a) and (c) show the number of pixels within the fields shown in Figure 2.

and 8, which decreases the ratio of foliage area to ground area, yielding a larger penetration into canopy. Additionally, we also conclude that the ratio of $\mathrm{HH}$ and $\mathrm{VV}$ backscattering converges to 1 for field 7 and 8 as a result of distributed large amount of panicles, grains and leaves over the canopy surface. This heavy canopy top structure paved the way for better accuracy. The mean backscattering analysis of the fields - summarised in Figure 5- confirms our result about the diversity of morphological properties among the fields. It can be easily seen that small morphological differences were causes of backscatter variation, even though the fields were in all late reproductive stages. It is also to be noticed that the fields with the lowest standard deviation of backscattering are associated with the fields having the highest absolute accuracy, such as fields 8, 7 and 5 (see, Figure 5(a)), underlying the importance of a canopy top layer homogeneity in InSAR based crop height measurements. 
Table 3: Interferometric height measurements from September 2014

\begin{tabular}{cccccccc}
\hline & \multicolumn{3}{c}{$\mathrm{HH}[\mathrm{cm}]$} & & \multicolumn{3}{c}{$\mathrm{VV}[\mathrm{cm}]$} \\
field \# & $h_{H H}$ & $\sigma_{h_{H H}}$ & $\overline{\Delta h}$ & & $h_{V V}$ & $\sigma_{h_{V V}}$ & $\overline{\Delta h}_{V V}$ \\
\hline & & & & & & \\
1 & 17 & 94 & 87 & & 65 & 97 & 40 \\
2 & 34 & 96 & 81 & & 69 & 92 & 46 \\
3 & 51 & 100 & 60 & & 73 & 97 & 38 \\
4 & 40 & 84 & 63 & & 60 & 81 & 42 \\
5 & 68 & 92 & 49 & & 84 & 90 & 33 \\
6 & 45 & 83 & 48 & & 55 & 80 & 37 \\
7 & 79 & 100 & 36 & & 105 & 95 & 10 \\
8 & 88 & 117 & 21 & & 92 & 108 & 17 \\
\hline
\end{tabular}

Table 4: Interferometric height measurements from September 2015

\begin{tabular}{cccccccc}
\hline & \multicolumn{3}{c}{$\mathrm{HH}[\mathrm{cm}]$} & & \multicolumn{3}{c}{$\mathrm{VV}[\mathrm{cm}]$} \\
field \# & $h_{H H}$ & $\sigma_{h_{H H}}$ & $\overline{\Delta h}$ & & $h_{V V}$ & $\sigma_{h_{V V}}$ & $\overline{\Delta h}_{V V}$ \\
\hline & & & & & & \\
1 & 91 & 45 & 19 & & 104 & 45 & 6 \\
2 & 90 & 49 & 29 & & 92 & 49 & 27 \\
3 & 87 & 46 & 17 & & 95 & 46 & 9 \\
4 & 67 & 53 & 38 & & 81 & 53 & 24 \\
5 & 104 & 65 & 15 & & 105 & 65 & 14 \\
\hline
\end{tabular}

Having analysed the September 2015 results shown in Figure 9(c)-(d), it was noticed that discrepancy of height measurements within and among the fields were very low in comparison with the 2014 results, making the increase in height estimation performance evident. As the figure reveals, the measurements with high uncertainty were not apparent in the 2015 dataset based on the decreasing $h_{2 \pi}$. Table 4 quantifies the 2015 performance. The generated crop height measurements showed a better accuracy than the 2014 measurements as summarised in Table 3. As in the 2014 case, VV crop height measurements have better absolute accuracy than the $\mathrm{HH}$ one. The most accurate fields such as 1,3 and 5 are the fields having the 
lowest standard deviation of backscattering as in Figure 5(b). This finding also corresponds well with the results from September 2014.

\subsection{PollnSAR-based crop height estimations}

As shown by Kugler et al. (2014) and Lee and Fatoyinbo (2015), PollnSAR-based height inversion has been successfully tested with TanDEM-X data in forestry studies, but due to the small baseline acquisitions -like the one in September 2014-, it was not convenient for agricultural purposes. Recent large baseline TanDEM-X acquisitions have made a crop height estimation by PollnSAR possible. An analysis of the dependency on baseline length for TanDEM-X acquisitions is analysed in depth by Kugler et al. (2015), with similar sensitivity analysis published in (Cloude, 2010) and (Lopez-Sanchez and Ballester-Berman, 2009). As it was stated by Cloude (2010), the $\kappa_{v}$ parameter, defined as $\kappa_{v}=\kappa_{Z} h_{v} / 2$ should be ideally in the range 1-1.25 to provide an optimum estimation of vegetation height by PollnSAR. That optimum range comes from a trade-off between the simultaneous requirements of high coherence and enough volume decorrelation to provide sensitivity. In our case, considering an average vegetation height around $1 \mathrm{~m}$, the $\kappa_{v}$ values for the acquisitions in September 2014 and 2015 are 0.06 and 0.5, respectively. Therefore, crop height estimation is assessed with only September 2015 images, for which $\kappa_{v}$ is much closer to the required value. Regarding the preprocessing of the data, the size of the speckle filter was $15 \times 15$ in this case, since the number of looks required for estimation of the PollnSAR matrix is much larger than for the other approaches.

There exist different approaches to invert vegetation height from PollnSAR data according to the previous expressions. In this work we base the inversion on the algorithm described in (Kugler et al., 2014) also for TanDEM-X data, so readers are referred to that paper for details. The main steps are:

1. Line fit to a set of coherences or coherence region: estimation of the coherences with maximum and minimum ground contributions: $\tilde{\gamma}_{v o l}\left(\kappa_{Z}, \vec{w}_{\text {max }}\right)$ and $\tilde{\gamma}_{v o l}\left(\kappa_{Z}, \vec{w}_{\text {min }}\right)$.

2. SNR correction of the two complex coherences corresponding to the maximum and minimum ground contributions.

3. Estimation of the topographic phase $\phi_{0}$.

4. Estimation of vegetation height $h_{v}$.

A couple of differences with respect to that method are considered here to adapt it to our problem. First, in the case of a dominant double-bounce from the ground, as in equation (7), the extra decorrelation term shifts the topographic phase with respect to the point at which the line crosses the unit circumference, as it can be observed when $m_{D B}$ tends to infinity. Second, instead of inverting the whole model (i.e. all the parameters: height, extinction and ground-to-volume ratios) we focus our efforts in retrieving only the vegetation height $h_{v}$, as it was suggested in Cloude (2010), since it is the variable of interest for this work. Therefore, we employed a simplified expression proposed by (Cloude, 2010, Eq. 8.38) to derive the height:

$$
h_{v}=\left[\arg \left(\gamma_{v} e^{-i \phi_{0}}\right)+\eta\left(\pi-2 \arcsin \left(\left|\gamma_{v}\right|^{0.8}\right)\right)\right] / k_{z}
$$


where $\gamma_{v}$ corresponds to the coherence at a channel without any ground contribution, i.e. $\tilde{\gamma}_{v o l}\left(\kappa_{Z}, \vec{w}_{\min }\right)$, and $\eta$ is an adjustment parameter. The role of $\eta$ is to provide a balance or trade-off between the two types of information contained in interferometric data: amplitude (coherence) and phase. Depending on the reliability of them, one can choose a different value of eta (between 0 and 1) to provide height estimates in which the influence of errors in the estimation of coherence and phase is lowered or compensated. In this work we used $\eta=0.2$ for all results, since it made the retrieved heights to fall inside the correct range.

To cope with the mentioned shift in the topographic phase in a simple way, we use one iteration of the last two steps. First we assume $\phi_{0}$ is not affected by that shift and obtain a first estimate of it and of $h_{v}$. Then we use that value of $h_{v}$ to correct the topographic phase and, finally, $h_{v}$ is estimated again with equation (11). This simple approach is valid because for our data the product of the vertical wavenumber times the crop height is not large and, hence, the extra decorrelation term produced by the dominant double-bounce does not go below 0.98 . Consequently, both the topographic phase and the height employed as initial guess (which come from assuming a unit value for that term) are very close to the actual values. Then, the first iteration provides a small refinement which is enough (as the results show afterwards) for the scope of this work.

Fig. 10 illustrates the boundary of coherence values $\tilde{\gamma}\left(\kappa_{Z}, \vec{w}\right)$ with varying polarization $w_{i}$ on the complex unitary circle for each monitored rice cultivated field. $0 \leq\left|\tilde{\gamma}\left(\kappa_{Z}, \vec{w}\right)\right| \leq 1$ and $\arg \left\{\tilde{\gamma}\left(\kappa_{Z}, \vec{w}\right)\right\}$ provide the absolute value of the coherence (radial distance) and the scattering phase center (polar angle), respectively. The absolute location of the ellipses depends on the phase difference with respect to the reference DEM employed in the processing. However, this absolute location does not affect the procedure of height estimation based on PollnSAR because the retrieval of height is relative to the ground phase estimated by the algorithm itself, not relative to the reference DEM. The shape of the ellipses show how the complex coherence values change according to the polarisation channel. Polarimetric diversity, i.e. a polarisation-dependent difference in the position of the coherences as a function of the polarimetric channel, is a useful indicator of volume scattering decorrelation, making the PollnSAR inversion feasible.

Crop height estimations for each field with PollnSAR inversion are visualised in Figure 11 and briefly summarised in Table 5, where $h_{v}, s_{h_{v}}$ and $\overline{\Delta h}$ represent the mean crop height measurement, its sample standard deviation and the difference between the crop height measurements from PollnSAR and the insitu measurements conducted on August 28, 2015, respectively. In the particular case of PollnSAR the parameter that is estimated as vegetation height is the vertical length of the vegetation volume that is above the ground level. For flooded rice fields this ground level corresponds to the water surface level. Consequently, the comparison of the estimates is carried out against the height above water values that were measured in situ (see Section 3). In contrast to the InSAR-based estimation, which is obtained by subtracting a reference height obtained at a different (vegetation free) date, here the estimation is computed from a single date acquisition.

It can be easily seen that there is a consistency in the accuracy of the measurements among and within 


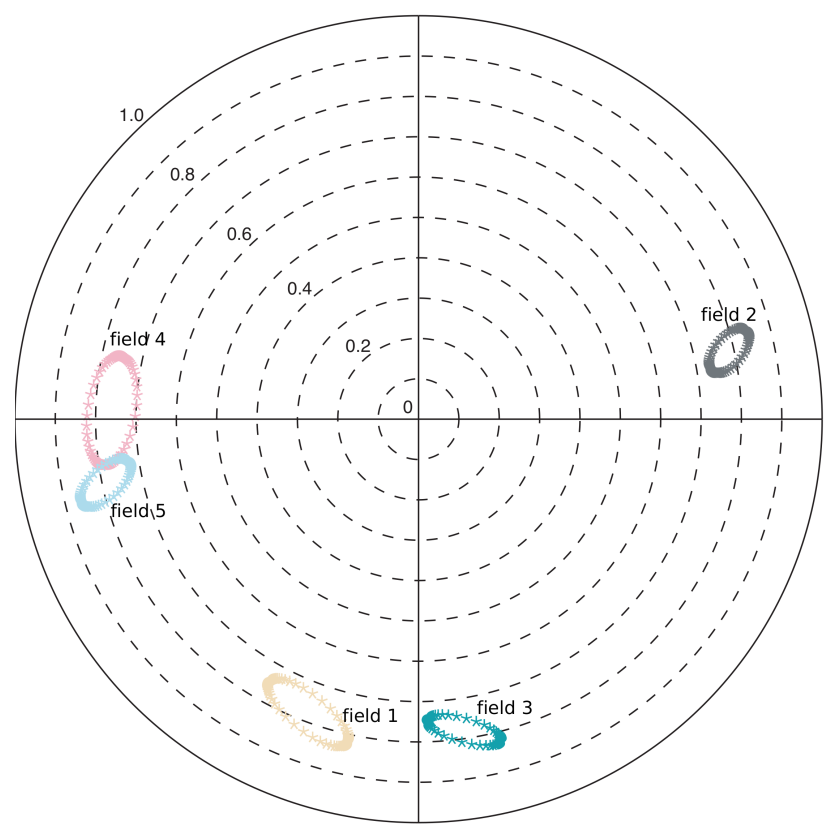

Figure 10: The boundary of coherence region, formed by varying polarisation, for each rice cultivated fields at the date of acquisition.

Table 5: PollnSAR height measurements $\mathrm{h}_{\mathrm{v}}$ from September 2015

\begin{tabular}{cccc} 
field \# & $h_{v}$ & $s_{h_{v}}$ & $\overline{\Delta h}$ \\
\hline 1 & 98 & 9 & -2 \\
2 & 95 & 20 & 11 \\
3 & 108 & 14 & -15 \\
4 & 87 & 9 & 15 \\
5 & 103 & 19 & 15 \\
\hline
\end{tabular}

the fields. The minimum absolute accuracy has turned out to be about $2 \mathrm{~cm}$ for field 1 . However, there are offsets greater than $10 \mathrm{~cm}$ with respect to the ground truth for the other fields, which can be caused by several reasons. First, the specific model used for the inversion is extremely simple and may overlook some of the features present in the rice fields, like differences in the attenuation at different polarimetric channels. Second, a larger baseline would be really required to ensure working in the best conditions, as indicated by the small value of $\kappa_{v}$ with respect to the ideal case. However, the absolute accuracy values ranging from 2 to $15 \mathrm{~cm}$ are of interest for vegetation height monitoring from space and thus show that PollnSAR inversion may be able to meet the requirement of global canopy monitoring with increasing spatial baseline in terms of bi-static space-based acquisition mode.

To sum up, with regards to the large baseline and bi-static polarimetric acquisition mode, TanDEM-X 


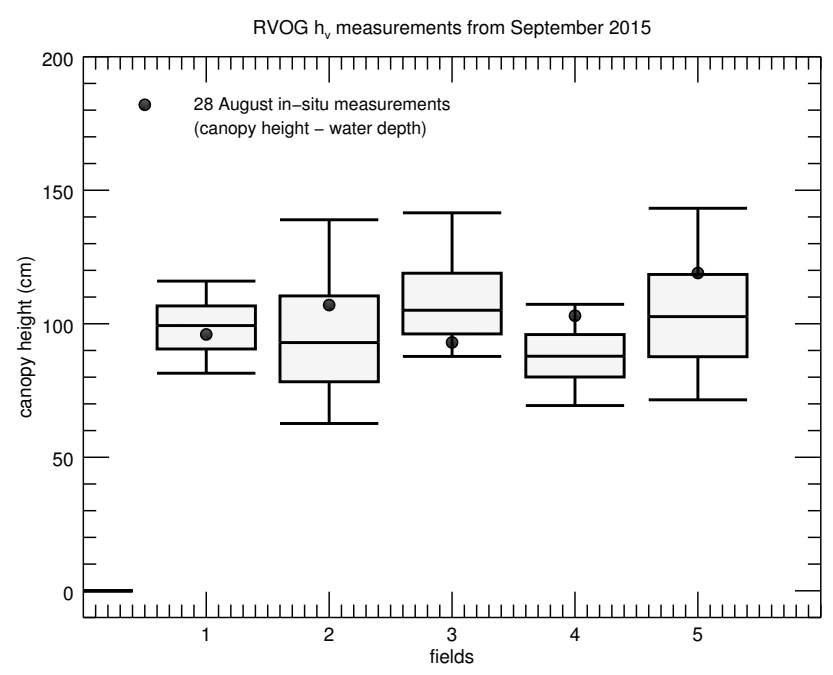

Figure 11: Retrieved vegetation height $h_{v}$ from PollnSAR (11). The solid circle refers to the in situ measured height.

images acquired on September 5, 2015 were used for crop height estimation by using backscattering, InSAR and PollnSAR together. Table 2, 4 and 5 summarise the absolute crop height estimations from each field with regards to the in-situ measurements. $\overline{\Delta h}$ values from backscattering and PollnSAR inversions were very consistent each other, irrespective of the conditions of the fields. However, the behaviour of interferometric measurements among the fields was slightly different -as expected- than the other two methods. It is to be noticed that there is an influence of using external DEM (vegetation free) on the absolute accuracy results of InSAR-based measurements.

The estimated 2015 root mean square errors (RMSE) for PollnSAR, metamodel-driven morphological backscattering model and InSAR (VV) were 12, 13 and $18 \mathrm{~cm}$, respectively. For the 2014 dataset, RMSEs of 8 and $35 \mathrm{~cm}$ were obtained by backscattering and InSAR (VV) based measurements, respectively. The RMSE of 2014 backscattering based approach has statistics comparable with the 2015 one, with a slightly better $(\sim 5 \mathrm{~cm})$ estimation. However, for the InSAR based measurements, the RMSE values change significantly $(\sim 17 \mathrm{~cm})$ depending on the baseline configuration of the images.

\section{Summary and conclusions}

This work presents crop height estimation from space-based SAR sensors, specifically TanDEM-X, using backscattering, InSAR and PollnSAR inversion methods for underpinning step towards the development of a fully automatic monitoring system. The assessment of the methods has been illustrated with paddy rice by comparing in-situ measurements conducted during the reproductive seasons of 2014 and 2015. With these two independent year acquisitions, it has been possible to identify the optimal conditions for each method through temporally changing spatial baseline.

Decomposing the total backscattering $\sigma^{0}$ into crop morphological parameters requires computationally expensive simulations. To reduce the computational time and the complexity involved in the crop height 
estimation from $\sigma^{0}$, a metamodel is implemented. Applying a metamodel-driven morphological backscattering model, the crop heights of paddy rice grown in the test fields were estimated by taking into account $\mathrm{HH}$ and VV polarised acquisitions together. Based on the crop height in-situ measurements, absolute errors ranging between 3 and $22 \mathrm{~cm}$ were estimated. The mean absolute error for all fields is 9 $\mathrm{cm}$, with a standard deviation of $6 \mathrm{~cm}$. It has been shown that the metamodel based crop height inversion by backscattering allows a fast and adequate crop height information. Using metamodel significantly reduces the time and effort required to simulate RTT based models in precision agriculture (large-scale agricultural lands). Being also independent of spatial baseline configuration, the metamodel based RTT approach provides consistent results in both years and appears to be a feasible choice for crop monitoring. Although the integration of SAR measurements and agricultural principles provides not only crop height but also detailed biophysical information of crops, the precision of the RTT based backscattering model needs to be further investigated for operational monitoring including other crops differ from rice in terms of biophysical characteristics. The main limitation of the metamodel-driven morphological backscattering model is the requirement of the pre-identification of the coarse growth stage to reduce the size of the solution space in the inversion scheme.

The crop height estimation by coherent (interferometric) methods, in contrast to the incoherent backscattering model, is very sensible to the system geometry. The dual-pol TanDEM-X images were acquired with different heights of ambiguity for the two years. By using interferometric principles, the canopy heights for each field were calculated using the difference between surface elevation information between September (with vegetation) and November (vegetation-free) TanDEM-X DEMs. A detailed comparison according to the in-situ measurements revealed that the absolute accuracy of the crop height measurements deteriorated with decreasing spatial baseline, as expected. Indeed, while a baseline of 102 $\mathrm{m}$ yielded a maximum absolute error of $46 \mathrm{~cm}$ in VV polarisation, this value strongly decreased to $27 \mathrm{~cm}$ with a spatial baseline of $932 \mathrm{~m}$.

In general, bi-static interferometric measurements can be used for the plant height monitoring by just using the phase differences between the acquisitions instead of the complex model characterising the interaction between canopy and the signal. Moreover, there is no need to integrate agronomical growth principles for interpreting the results, which makes InSAR-based methods advantageous in practical applications. Three main considerations can be drawn for this technique. First, the result accuracy has been demonstrated highly dependent on the spatial baseline: the larger the baseline, the more accurate the plant height estimation. Second, the architecture of the monitored crops, impacting on the resulting scattering phase centres, has been shown affecting the absolute accuracy of the polarised measurements. Here, higher coherence does not imply a better absolute accuracy. The absolute accuracy of the crop height measurements were better for VV polarised acquisitions due to the scattering being located closer to the top of the vegetation, since crops were in the reproductive stage. Third, an external terrain model, or a vegetation-free DEM, is required to derive the plant height. This can be seen as a disadvantage of the 
interferometric approach in terms of operational crop monitoring.

Finally, the inverse problem of determining the crop height is addressed with PollnSAR. The feasibility of PollnSAR approaches is limited by a non-volumetric decorrelation based on spatial baseline. Consequently, the 2014 acquisition was not considered. However, exploiting the 2015 acquisition, an impressive absolute height accuracy of $2 \mathrm{~cm}$ was obtained for one of the rice cultivated field. To our knowledge, the PollnSAR approach is for the first time used to estimate the height of the agricultural crops with space-based data. In this context, with its dual-pol acquisition capability, height estimation with TanDEM-X turned out to be reliable not only for forest height but also for crop height monitoring. A range of 2 to $15 \mathrm{~cm}$ absolute errors were measured by PollnSAR inversion when the fields were in the reproductive stage. Unlike the interferometric and backscattering inversions, the PollnSAR approach does not require neither an additional DEM nor advanced EM model with agronomical growth relations. Although very promising results have been reported with a very small height of ambiguity, the PollnSAR inversion needs further research with regard to a wide range of vegetation heights (e.g., 5 to $130 \mathrm{~cm}$ ).

In a nutshell, this study focused on the comparison and evaluation of crop height retrieval methods for microwave remote sensing applications, concentrating on X-band acquisitions. As summarised in Table 6, advantages and limitations of the three aforementioned methods have been listed, especially the requirements to be fulfilled for obtaining consistent crop height estimates. Further analysis needs to take into account a combination of backscattering and PollnSAR inversion models. They can strengthen each other to overcome their limitations in terms of farming monitoring, i.e., having a plant height from PollnSAR inversion will eliminate the requirement of the pre-identification of the coarse growth stage in backscattering inversion and will supply not only more reliable crop height estimation and but also other morphological parameters of the crops.

Table 6: Summary of the characteristics, pros and cons of the SAR related crop height estimation methods

\begin{tabular}{|r||c|c|c|c|}
\hline & $\begin{array}{c}\text { minimum required } \\
\text { acquisitions }\end{array}$ & external requirements & advantages & disadvantages \\
\hline \hline RTT & one single polarized & $\begin{array}{c}\text { EM model, crop growth } \\
\text { rules }\end{array}$ & considers full morphology & $\begin{array}{c}\text { advanced morphology } \\
\text { based EM needed }\end{array}$ \\
\hline \multirow{2}{*}{ InSAR } & two same polarized & vegetation-free DEM & deterministic & $\begin{array}{c}\text { short wavelength, } \\
\text { external DEM and bistatic } \\
\text { acquisition needed }\end{array}$ \\
\hline PollnSAR & two dual polarized & - & no additional data needed & large baseline needed \\
\hline
\end{tabular}

\section{Acknowledgment}

This work has been supported by the Scientific and Technological Research Council of Turkey (TUBITAK) under project 113Y446, by the Spanish Ministry of Economy and Competitiveness (MINECO) and EU FEDER under projects TEC2011-28201-C02-02 and TIN2014-55413-C2-2-P, and by the Scientific 
Research Projects Coordination of Istanbul Technical University (ITU-BAP) under project ID 37496.

\section{References}

Abdullahi, S., Kugler, F. \& Pretzsch, H. (2016). Prediction of stem volume in complex temperate forest stands using TanDEM-X SAR data. Remote Sensing of Environment, 174, 197-211. doi:10.1016/j.rse.2015.12.012

Ballester-Berman, J. D. \& Lopez-Sanchez, J. M. (2011). Combination of direct and double-bounce ground responses in the homogeneous oriented volume over ground model. IEEE Geoscience and Remote Sensing Letters, 8(1), 54-58.

Bamler, R. \& Hartl, P., (1998). Synthetic aperture radar interferometry. Inverse Problems, 14(4), 1-54.

Cloude, S. R. (2010). Polarisation: applications in remote sensing. Oxford University Press.

Engdahl, M. E., Borgeaud, M. \& Rast, M. (2001). The use of ERS-1/2 Tandem interferometric coherence in the estimation of agricultural crop heights. Geoscience and Remote Sensing, IEEE Transactions on, 39(8),1799-1806.

Erten, E. (2013). Glacier velocity estimation by means of a polarimetric similarity measure. Geoscience and Remote Sensing, IEEE Transactions on, 51(6), 3319-3327. doi:10.1109/TGRS.2012.2219873

Erten, E., Rossi, C. \& Yuzugullu, O. (2015). Polarization impact in TanDEM-X data over vertical oriented vegetation: the paddy-rice case study. IEEE Geoscience and Remote Sensing Letters, 12(7), 1501-1505. doi:10.1109/LGRS.2015.2410339

Guo, H., Liu, G., Liao, J., Li, X., Zhang, L., Shen, G., Fu, W. \& Sun, Z. (2014). Study of RADARSAT-2 synthetic aperture radar data for observing sensitive factors of global environmental change. Journal of Applied Remote Sensing, 8(1), 1-18.

Hanssen F. R., (2001). Radar interferometry: data interpretation and error analysis. Kluwer Academic Publishers.

Inoue, Y., Sakaiya, E. \& Wang, C. (2014). Potential of X-band images from high-resolution satellite SAR sensors to assess growth and yield in paddy rice. Remote Sensing, 6, 5995-6019.

Karam, M., Fung, A. \& Antar, Y. 1988. Electromagnetic wave scattering from some vegetation samples. Geoscience and Remote Sensing, IEEE Transactions on, 26(6), 799-808.

Karam, M. A., Amar, F., Fung, A. K., Mougin, E., Lopes, A., Le Vine, D. M. \& Beaudoin, A. (1995). A microwave polarimetric scattering model for forest canopies based on vector radiative transfer theory. Remote Sensing of Environment, 53(1),16-30. 
Karam, M. A., Fung, A. K., Lang, R. H. \& Chauhan, N. S. (1992). A microwave scattering model for layered vegetation. Geoscience and Remote Sensing, IEEE Transactions on, 30(4), 767-784.

Karila, K., Vastaranta, M., Karjalainen, M. \& Kaasalainen, S. (2015). TanDEM-X interferometry in the prediction of forest inventory attributes in managed boreal forests. Remote Sensing of Environment,159, 259-268. doi:10.1016/j.rse.2015.12.012

Kennedy, J., Ebehart, R. C. \& Shi, Y. (2001). Swarm intelligence. A volume in The Morgan Kaufmann Series in Artificial Intelligence, Chapter 7, 287-325.

Kim, Y., Lee, H. \& Hong, S. (2013). Continuous monitoring of rice growth with a stable ground-based scatterometer system. IEEE Geoscience and Remote Sensing Letters, 10(4), 831-835.

Koay, J. Y., Tan, C. P., Lim, K. S., bin Abu Bakar, S. B., Ewe H.T., Chuah, H.T. \& Kong, J. A. (2007). Paddy fields as electrically dense media: Theoretical modeling and measurement comparisons. Geoscience and Remote Sensing, IEEE Transactions on, 45(9), 2837-2849.

Kriger, G., Moreira, A., Fiedler, H., Hajnsek, I., Werner, M., Younis, M. \& Zink, M. (2007). TanDEM$\mathrm{X}: \mathrm{A}$ satellite formation for high-resolution SAR interferometry. Geoscience and Remote Sensing, IEEE Transactions on, 45(11), 3317-3341.

Kugler, F., Lee, S. K., Hajnsek, I. \& Papathanassiou, K. (2015). Forest height estimation by means of PolInSAR data inversion: The role of the vertical wavenumber. Geoscience and Remote Sensing, IEEE Transactions on, 53(10), 5294-5311.

Kugler, F., Schulze, D., Hajnsek, I., Pretzsch, H. \& Papathanassiou, K. (2014). TanDEM-X Pol-InSAR performance for forest height estimation. Geoscience and Remote Sensing, IEEE Transactions on, 52(10), 6404-6422.

Lancashire, P. D., Bleiholder, H., Langeluddecke, P., Stauss, R., van den Boom, T., Weber, E. \& WitzenBerger, A. (1991). A uniform decimal code for growth stages of crops and weeds. Annals of Applied Biology, 119(3), 561-601. doi:10.1111/j.1744-7348.1991.tb04895.x

Le Toan, T., Ribbes, F., Wang, L. F., Floury, N., Ding, K. H., Kong, J. A., Fujita, M. \& Kurosu, T. (1997). Rice crop mapping and monitoring using ERS-1 data based on experiment and modeling results. Geoscience and Remote Sensing, IEEE Transactions on, 35(1), 41-56.

Lee, S. K. \& Fatoyinbo, T. E. (2015). TanDEM-X Pol-InSAR inversion for mangrove canopy height estimation. Selected Topics in Applied Earth Observations and Remote Sensing, IEEE Journal of, 8(7), 3608-3618.

Li, Z., Guo, M., Wang, Z. Q. \& Zhao, L. (2014). Forest-height inversion using repeat-pass spaceborne PollnSAR data. Science China Earth Sciences, 57(6). 
Liu, Y., Chen, K. S., Xu, P. \& Li, Z. L. (2016). Modeling and characteristics of microwave backscattering from rice canopy over growth stages. Geoscience and Remote Sensing, IEEE Transactions on, doi:10.1109/TGRS.2016.2590439

Lopez-Sanchez, J. M. \& Ballester-Berman, J. D. (2009). Potentials of polarimetric SAR interferometry for agriculture monitoring. Radio Science, 44(2).

Lopez-Sanchez, J. M., Hajnsek, I. \& Ballester-Berman, J. D. (2012). First demonstration of agriculture height retrieval with PollnSAR airborne data. IEEE Geoscience and Remote Sensing Letters, 9(2), 242-246.

Marelli, S. \& Sudret, B. (2014). UQLab: a framework for uncertainty quantification in MATLAB. ETH-Zürich, DOI:10.1061/9780784413609.257.

Mason, D. C., Trigg, M., Garcia-Pintado, J., Cloke, H. L., Neal, J. C. \& Bates, P. D. (2016). Improving the TanDEM-X digital elevation model for flood modelling using flood extents from synthetic aperture radar images. Remote Sensing of Environment, 173, 15-28.

Minh, D. H. T., Tebaldini, S., Rocca, F., Le Toan, T., Villard, L., Dubois-Fernandez, P. C. (2015). Capabilities of BIOMASS tomography for investigating tropical forests. Geoscience and Remote Sensing, IEEE Transactions on, 53 (2), 965-975.

Patel, P. \& Srivastava, H. S. (2013). Ground truth planning for synthetic aperture radar (SAR): addressing various challenges using statistical approach. International Journal of Advancement in Remote Sensing, GIS, Geography, 1(2), 293-305.

Patel, P., Srivastava, H. S., Panigrahy, S. \& Parihar, J. S. (2006). Comparative evaluation of the sensitivity of multi-polarized multi-frequency SAR backscatter to plant density. International Journal of Remote Sensing, 27(2), 293-305.

Pichierri, M., Hajnsek, I. \& Konstantinos, P. (2016). A multibaseline Pol-InSAR inversion scheme for crop parameter estimation at different frequencies. Geoscience and Remote Sensing, IEEE Transactions on, 54(8), 4952-4970. doi:10.1109/TGRS.2016.2553739

Rajabi, M. M., Ataie-Ashtiani, B. \& Simmons, C. T. (2015). Polynomial chaos expansions for uncertainty propagation and moment independent sensitivity analysis of seawater intrusion simulations. Journal of Hydrology, 520, 101-122.

Rizzoli, P., Brautigam, B., Kraus, T., Martone, M. \& Krieger, G. (2012). Relative height error analysis of TanDEM-X elevation data. ISPRS Journal of Photogrammetry and Remote Sensing, 73, 30-38.

Romeiser, R. and Runge, H. and Suchandt, S. and Kahle, R. \& Bell, P. S. (2014). Quality Assessment of Surface Current Fields From TerraSAR-X and TanDEM-X Along-Track Interferometry and Doppler 
Centroid Analysis. Geoscience and Remote Sensing, IEEE Transactions on, 52(5), 2759-2772. doi:10.1109/TGRS.2013.2265659

Rossi, C. \& Erten, E. 2015. Paddy-rice monitoring using TanDEM-X. Geoscience and Remote Sensing, IEEE Transactions on, 53(2), 900-910. doi:10.1109/TGRS.2014.2330377

Rossi, C., Gonzalez, F. R., Fritz, T., Yague-Martinez, N. \& Eineder, M. (2012). TanDEM-X calibrated raw DEM generation. ISPRS Journal of Photogrammetry and Remote Sensing, 73, 12-20. http://dx.doi.org/10.1016/j.isprsjprs.2012.05.014

Rossi, C., Minet, C., Fritz, T., Eineder, M. \& Bamler, R. (2016). Temporal monitoring of subglacial volcanoes with TanDEM-X -Application to the 2014-2015 eruption within the Bardarbunga volcanic system, Iceland. Remote Sensing of Environment, 181, 186-197. doi:10.1016/j.rse.2016.04.003

Sochala, P. \& Le Maitre, O. P. (2013). Polynomial chaos expansion for subsurface flows with uncertain soil parameters. Advances in Water Resources, 62(Part A), 139-154.

Soja, M., Persson, H. \& Ulander, L. (2015). Estimation of forest biomass from two-level model inversion of single pass InSAR data. Geoscience and Remote Sensing, IEEE Transactions on, 53(9), 5083-5099.

Srivastava, H. S., Patel. P. \& Navalgund, R. (2006). Application potentials of synthetic aperture radar interferometry for land-cover mapping and crop-height estimation. Current Science, 91(6), 783-786.

Srivastava, H. S., Patel, P., Prasad, S. N., Sharma, Y., and et al. (2007). Potential applications of multiparametric Synthetic Aperture Radar (SAR) data in wetland inventory: A case study of Keoladeo National Park (a world heritage and Ramsar site), Bharatpur, India. In Proceedings: Taal 2007: the 12th World Lake Conference, 1862-1879.

Srivastava, H. S., Patel, P., Sharma, Y. \& Navalgund, R. (2009). Multi-frequency and multipolarized SAR response to thin vegetation and scattered trees. Current Science, 97(3), 425-429.

Sudret, B., 2015. Polynomial chaos expansions and stochastic finite element methods, Chapter 6. Taylor and Francis.

Tanase, M., Panciera, R., Lowell, K., Tian, S., Garcia-Martin, A. \& Walker, J. (2014). Sensitivity of L-band radar backscatter to forest biomass in semiarid environments: A comparative analysis of parametric and nonparametric models. Geoscience and Remote Sensing, IEEE Transactions on, 52(8), 4671-4685.

Treuhaft, R. N., Madsen, S. N., Moghaddam, M. \& van Zyl, J. J. (1996). Vegetation characteristics and underlying topography from interferometric radar. Radio Science, 31(6), 1449-1485.

Treuhaft, R. N. \& Siqueira, P. R. (2000). Vertical structure of vegetated land surfaces from interferometric and polarimetric data. Radio Science, 35, 141-177. 
Vasko, A., Thirion-Lefevre, L., Bilicz, S., Champion, I., Lambert, M. \& Gyimothy, S. (2011). Metamodelbased adaptive use of a coherent polarimetric backscattering simulator for the characterization of forested areas at low frequencies. In proocedings: Electromagnetics Research Symposium (PIERS 2011), Suzhou, China, 818-821.

Vicente-Guijalba, F., Martinez-Marin, T., Lopez-Sanchez, J. M. (2015). Dynamical approach for real-time monitoring of agricultural crops. Geoscience and Remote Sensing, IEEE Transactions on, 53(6), 32783293.

Villard, L. \& Le Toan, T. (2015). Relating P-Band SAR intensity to biomass for tropical dense forests in hilly terrain: $\gamma^{0}$ or $t^{0}$ ? Selected Topics in Applied Earth Observations and Remote Sensing, IEEE Journal of, 8(1), 214-223.

Villard, L., Minh, H. T. \& Le Toan, T. (2015). PollnSAR height retrieval method based on electromagnetic forward meta-model initiated with TomoSAR estimates: Applications on TropiSAR data and prospects for biomass mission. In: 1st Biomass Science Workshop, ESA-ESRIN, Italy.

Wang, L. \& Kong, J. A. (2005). Electromagetic scattering model for rice canopy based on Monte Carlo simulation. Progress In Electromagnetics Research, 52, 153-171.

Wang, C., Wu, J., Zhang, Y., Pan, G., Qi, J. \& Salas, W. A. (2009). Characterising L-band scattering of paddy rice in southeast China with radiative transfer model and multitemporal ALOS/PALSAR imagery. Geoscience and Remote Sensing, IEEE Transactions on, 47(4), 988-998.

Yuzugullu, O., Marelli, S., Erten, E., Sudret, B. \& Hajnsek, I. (2015). Global sensitivity analysis of a morphology based electromagnetic scattering model. In proceedings: Geoscience and Remote Sensing Symposium (IGARSS), 2015 IEEE International, 1017-1020. doi:10.1109/IGARSS.2015.7326381

Yuzugullu, O., Marelli, S., Erten, E., Sudret, B. \& Hajnsek, I. (2016). A metamodel-based inversion for determining rice growth stage from SAR data. Remote Sensing of Environment, under review.

Yuzugullu, O., Erten, E. \& Hajnsek, I. (2016). Estimation of rice crop height from X- and C- band PolSAR by metamodel-based optimization. IEEE Journal of Selected Topics in Applied Earth Observations and Remote Sensing. doi: 10.1109/JSTARS.2016.2575362

Zalite, K., Antropov, O., Praks, J., Voormansik, K. \& Noorma, M. (2015). Monitoring of agricultural grasslands with time series of X-Band repeat-pass interferometric SAR. Selected Topics in Applied Earth Observations and Remote Sensing, IEEE Journal of, available online.

Zhang, S., Foerster, S., Medeiros, P., Carlos de Araujo, J., Motagh, M. \& Waske, B. (2016). Bathymetric survey of water reservoirs in north-eastern Brazil based on TanDEM-X satellite data. Science of The Total Environment, doi:10.1016/j.scitotenv.2016.07.024. 
774 Zhang, Y., Liu, X., Su, S. \& Wang, C. (2014). Retrieving canopy height and density of paddy rice from 775 Radarsat-2 images with a canopy scattering model. International Journal of Applied Earth Observation 776 and Geoinformation, 28, 170-180. 